\title{
ACCELERATED BONE GROWTH REMOTELY INDUCED BY MAGNETIC FIELDS AND SMART MATERIALS
}

\author{
UNDERGRADUATE HONORS THESIS
}

\author{
By \\ Stephen Andrew Hart \\ The Ohio State University \\ Department of Mechanical Engineering
}

19 May 2006

Approved By:

Dr. Marcelo J. Dapino, Advisor:

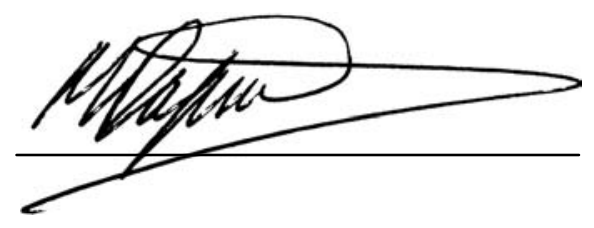


(C) Copyright by

Stephen Andrew Hart

2006 


\section{Table of Contents}

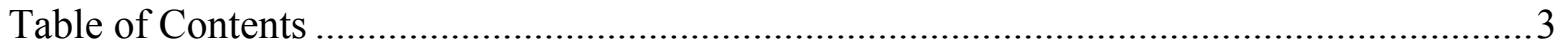

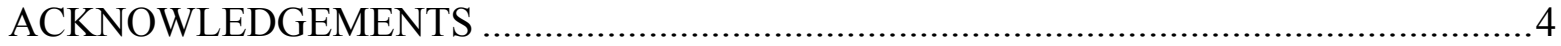

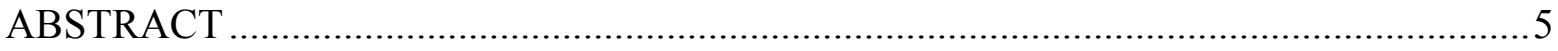

1. INTRODUCTION TO MECHANICALLY MEDIATED BONE GROWTH .............. 7

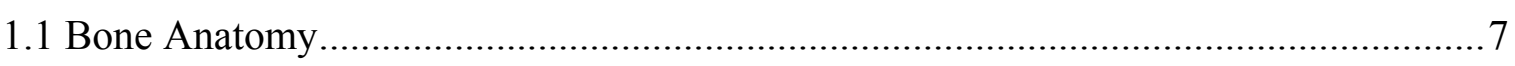

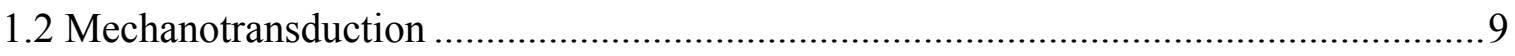

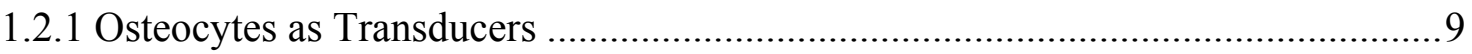

1.2.2 The Role of Strain in the Osteogenic Response ............................................. 10

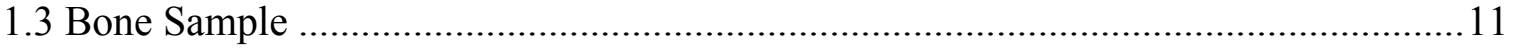

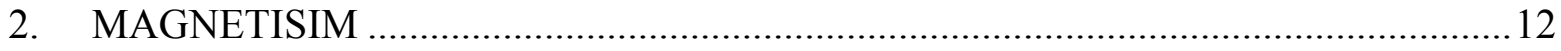

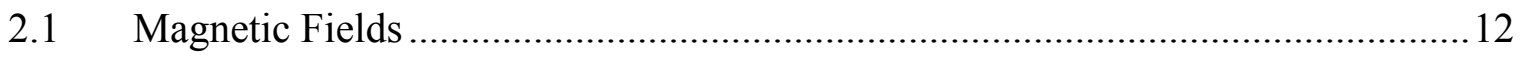

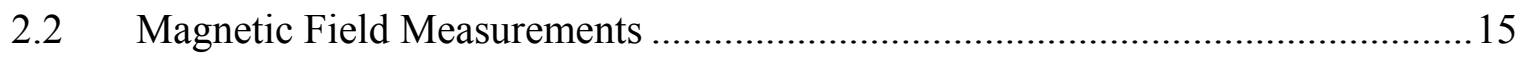

2.3 Characteristics of Magnetic Materials................................................................. 16

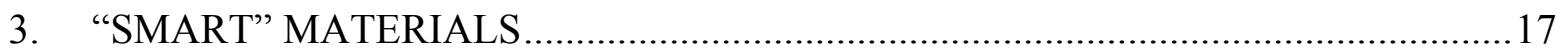

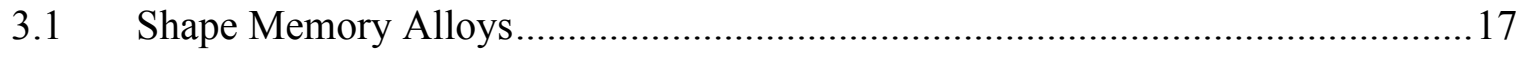

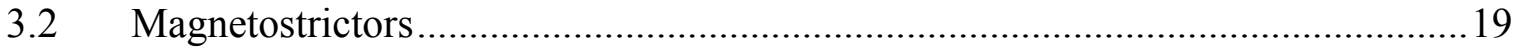

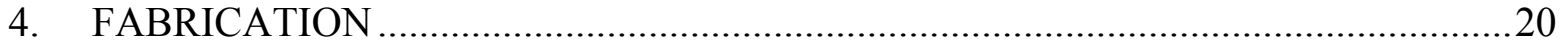

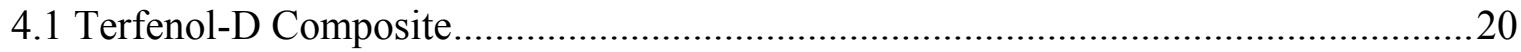

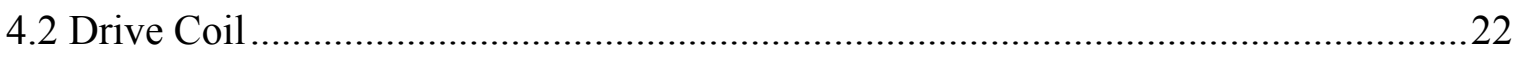

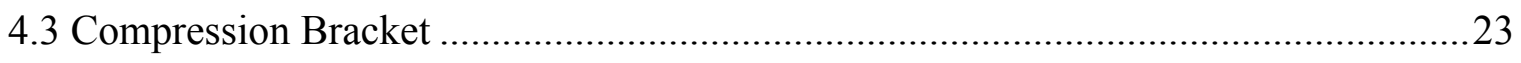

5. CHARACTERIZATION OF TERFENOL-D COMPOSITE ....................................24

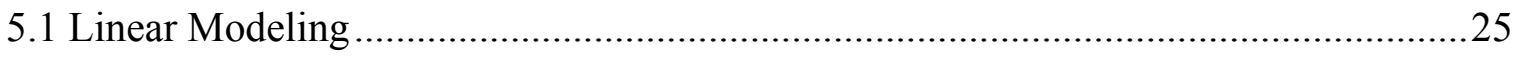

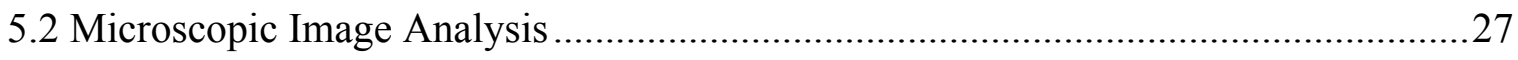

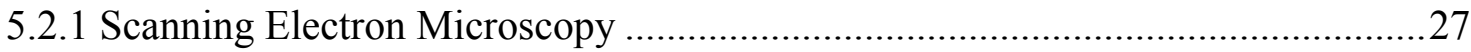

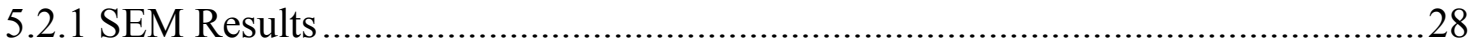

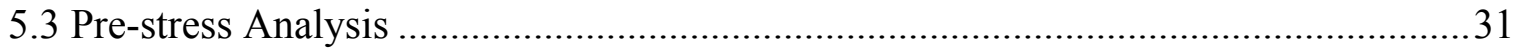

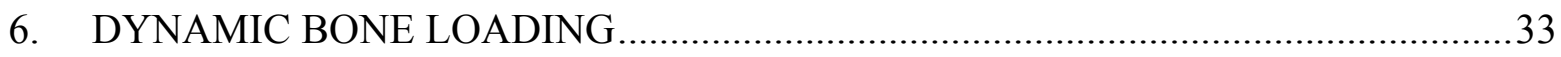

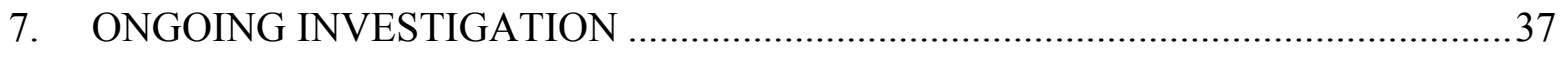

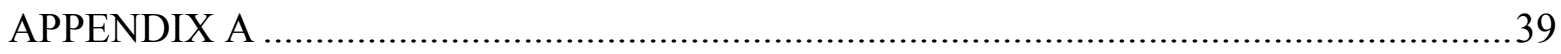

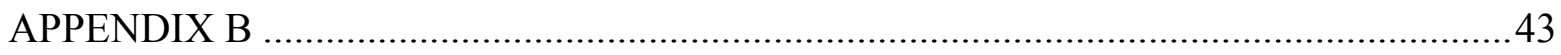

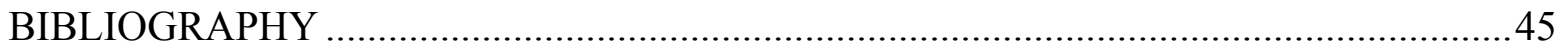




\section{ACKNOWLEDGEMENTS}

I would like to sincerely thank Prof. Marcelo Dapino for his continual advice, support, and encouragement without which this project would not have been completed. In addition, I would like to thank the other students in the Smart Materials and Structures Laboratory including Xiang, Phillip, Michael, and Neelesh for helping me with data acquisition trouble shooting, fabrication techniques, and general insight into the subject matter. I would also like to acknowledge Cameron Begg and Steve Bright at the Campus Electron Optics Facility for their assistance with the scanning electron microscopy investigation and to Dr. Glen Njus at the Calhoun Research Laboratory affiliated with the Akron University Department of Biomedical Engineering for providing the bone sample used in this research. Finally, I would like to extend a special thanks to the College of Engineering for providing the financial means to pursue this research. 


\section{ABSTRACT}

This paper presents the novel approach of employing active or "smart" materials to serve as mechanical stimuli for promoting bone growth. It has been shown that when bone is placed under oscillating bending stresses, it will adapt to better support the load and as a result, the bone will grow and strengthen. Furthermore, the strain stimulus induced in bone as a result of oscillating bending stresses is directly proportional to both the magnitude and frequency of the strain signal. Most current research is based on electromechanical or thermomechanical methods to produce bone stress. The resulting systems can be prohibitively large or require frequent surgery for adjustment. This proposal is focused on the creation of a smart materials-based bone-loading apparatus that could produce enough stress to promote bone growth while remaining compact and minimally invasive.

To satisfy these requirements, magnetostrictive compounds that deform in the presence of magnetic fields are considered. These materials have the ability to convert magnetic energy to mechanical energy and vise versa. Applied magnetic fields cause domains to rotate thus changing the overall shape of the material. One magnetostrictive material considered is Terfenol-D, an alloy of terbium, dysprosium, and iron. This alloy was chosen because it exhibits a high saturation strain, or maximum strain level attainable, relative to other magnetostrictive materials. To increase functionality and resilience of the alloy, Terfenol-D is used in composite form using micron-sized Terfenol-D particles embedded in an epoxy matrix magnetically aligned during the cure of the composite. To magnetically activate the sample, a solenoid was constructed with 2400 turns of 20 AWG magnet wire able to produce a maximum quasi-static field of $405 \mathrm{kA} / \mathrm{m}$.

Initial quasi-static tests of the composite were conducted with free-free boundary conditions to measure the maximum magnetostriction of the sample. Strain was measured by two strain gages, one on each of the axial surfaces of a half-cylinder composite sample of Terfenol-D. Tests were conducted by applying a $180 \mathrm{~V}$ sine wave signal at a frequency of $0.15 \mathrm{~Hz}$ to the solenoid. The maximum strain level produced by the sample was 575 microstrain at a maximum field of $300 \mathrm{kA} / \mathrm{m}$. Tests at $30 \mathrm{~Hz}$ were then conducted producing 2300 microstrain at a field of $170 \mathrm{kA} / \mathrm{m}$. 
The Terfenol-D composite was then bonded to the surface of a porcine tibia. The composite was driven at a frequency of $30 \mathrm{~Hz}$ and a field of $170 \mathrm{kA} / \mathrm{m}$. Test results showed that the strain production on the surface of the bone exceeded 1000 microstrain. This is sufficient strain magnitude and frequency to promote cortical bone growth in both rats and turkeys, and maintain cortical bone structure in humans. 


\section{INTRODUCTION TO MECHANICALLY MEDIATED BONE GROWTH}

Bone is a unique biological tissue that is lightweight, strong, and resilient. In addition to structural integrity, bone also has the remarkable ability to adapt to varying loading environments. In this way, bone is able to remodel itself to best support normal loading conditions. This leads to the intriguing idea that atypically applied bone loading could generate a desired bone growth pattern. To investigate bone growth, it is necessary to discuss the biological functioning of bone. To that end, an overview of long bone anatomy and physiology is provided. Many references are available for a complete review of bone histology and biomechanics $[1,10]$.

\subsection{Bone Anatomy}

The bones of the skeleton are broadly categorized into two subtypes. Flat bones comprise the axial skeleton and include bones such as the skull, vertebrae, and pelvis. Long bones, on the other hand, comprise the appendicular skeleton and include the bones of the limbs such as the femur in the thigh and ulna in the arm. These bones are characterized by having two extremities wider than the connecting shaft. Each extremity of a long bone is termed the epiphysis and the cylindrical shaft is termed the diaphysis. The region between the two is termed the metaphysis. 


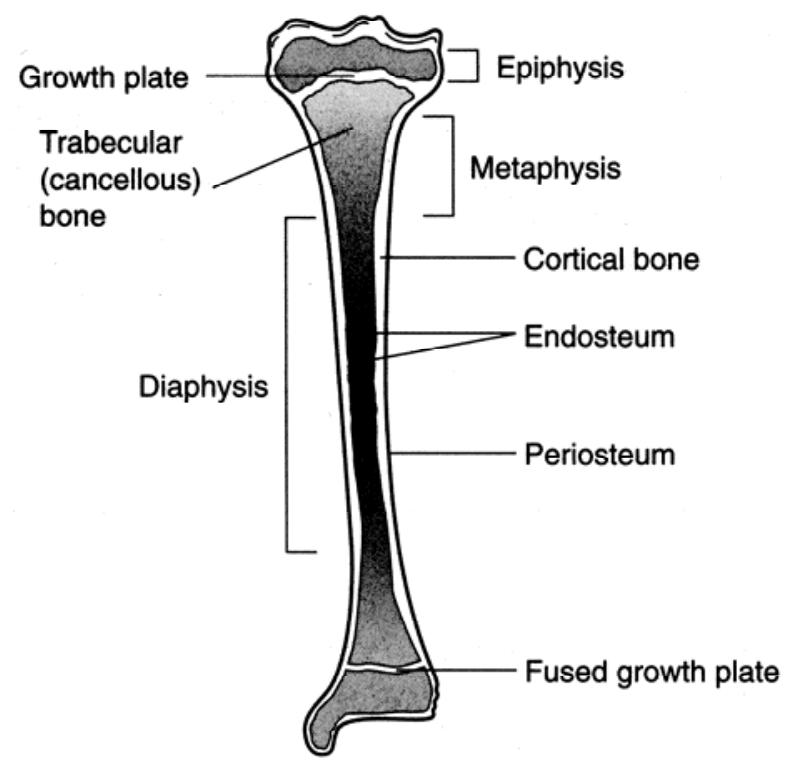

Figure 1.1: Long Bone Anatomy [10]

Microscopically, bone may also be categorized into two subtypes. Trabecular or cancellous bone is found mainly in the epiphysis but also the interior of the diaphysis of long bones. This type of bone consists of a porous network of fiber-like trabeculae. The porous architecture allows bone marrow, blood vessels, and metabolic materials to reach the load bearing portion of the bone, termed cortical bone. Cortical bone has a dense structure and surrounds the entire surface of the bone. Typically, it is thin around the epiphysis and thick through the diaphysis. The cortical bone through the diaphysis surrounds the bone marrow in the medullary cavity. The outer surface of this cortical bone is termed the periosteum and the inner surface is termed the endosteum. Mechanically induced bone growth, as will be described shortly, primarily occurs on these two surfaces.

On a cellular level, bone contains three important cell types. Osteoblasts are found on bone formation surfaces and are responsible for the mineralization of new bone. As bone growth accumulates, osteoblasts become buried by their own products and differentiate into osteocytes. The osteocytes reside within the mineralized bone in order to maintain the bone structure. Osteocytes, however, do not act as isolated cells. Instead, they are connected by long cellular processes to other osteocytes within the bone matrix and the osteoblasts on the bone formation surfaces. This is thought to permit intercellular communication [12]. Opposed to osteoblasts, osteoclasts absorb old bone material. During bone remodeling, 
osteoblast activity is linked to osteoclast activity [4]. This indicates that the dynamic nature of bone growth builds new bone while simultaneously removing old bone.

\subsection{Mechanotransduction}

Julius Wolff first postulated in 1892 that bone structure adapts to changing stress environments [33]. Knowledge of the exact mechanism of how bone changes to meet different demands, however, continues to remain elusive. A contemporary theory of bone response contends that bone attempts to maintain an optimum strain level on the bone formation surfaces and remodels in such a way to achieve this strain optimum at all times [28]. In addition to the homeostasis mechanism, it has been shown that strain magnitude, strain rate, strain distribution, and strain cycles may also be important parameters in the mechanical stimulus.

\subsubsection{Osteocytes as Transducers}

The prevailing theory on mechanotransduction states that osteocytes act as transducers from mechanical loading to biochemical response [1, 5, 28]. A long bone loaded in compression, a femur for example, will induce a bending stress through the length of the bone because of the natural curvature of long bones and eccentric loading on the femoral head. The bending stresses create pressure gradients across the cortical and trabeculae architecture of the diaphysis. As the bone is bent, the concave surface will be

placed in compression and the convex surface in tension. Fluid is subsequently "squeezed" from the region of compression to the region of tension as shown in Figure 1.2. 


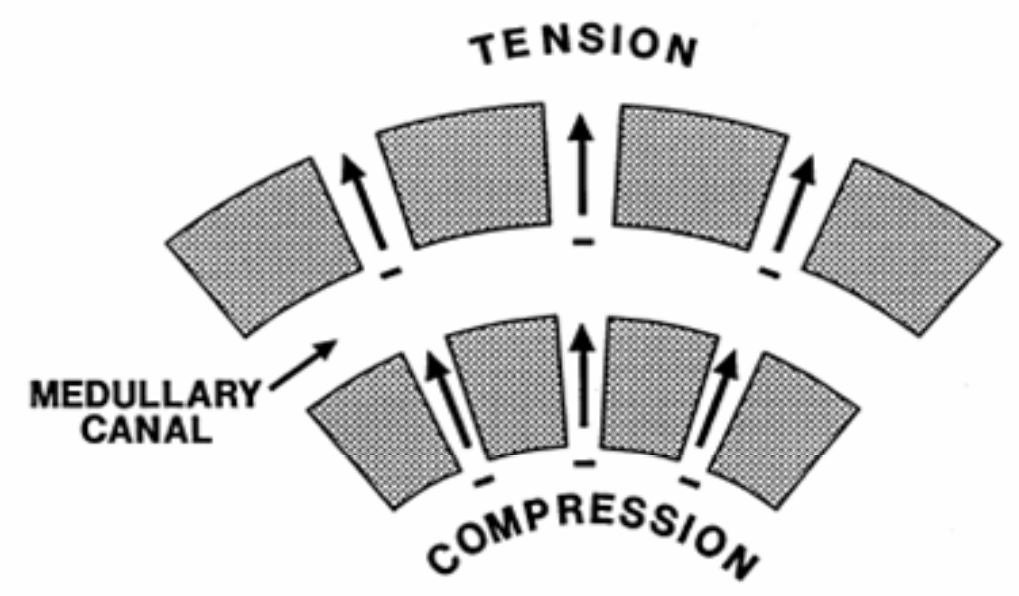

Figure 1.2: Diagram representing fluid flow in a stressed long bone

The fluid flow through the bone creates fluid shear within the bone which in turn promotes the anabolic response of growth [29]. If the bending moment is reversed, the fluid motion too would reverse and additional fluid shear would result. Although the exact mechanism is unclear, it has been shown that osteocytes, in addition to bone-lining cells, act as the transducers to oscillating fluid shear stress [1,5].

\subsubsection{The Role of Strain in the Osteogenic Response}

The first study that linked bone strain to bone growth observed a direct relationship between strain magnitude and new bone formation [19]. This observation was later developed into the optimum strain magnitude theory, also known as the error strain distribution theory [20]. This theory contends that unusual loading of the skeleton is the best promoter of osteogenesis. If unusual loading is repeated however, the osteogenic potential decreases as the "unusual" loading becomes customary.

In humans, strains below $200 \mu \varepsilon$ do not initiate any anabolic response in the cortical bone structure [7]. This level of loading is known as the trivial loading zone. However, low magnitude strain signals $(<200 \mu \varepsilon)$ have proven to promote trabecular bone structure $[18$, 21]. Strain values between 200 and $2000 \mu \varepsilon$ represent physiological levels of strain on the human skeleton. Osteoblast and osteoclast activity occurs in the physiological region of strain and ensures that bone continually renews itself. Above $2000 \mu \varepsilon$, the relative rate of bone formation to absorption increases and growth is observed [12]. For reference, $25000 \mu \varepsilon$ represents pathological strain magnitude in cortical bone structure. 
Contrary to the human case, studies involving turkeys have shown that a $30 \mathrm{~Hz}$ $200 \mu \varepsilon$ signal is sufficient to induce cortical bone growth [21]. Studies involving rats have shown that a $1050 \mu \varepsilon$ signal is sufficient to produce cortical bone growth [30].

Along with strain magnitude, the strain rate, or rate at which strain is applied and released, also plays an important role in new bone formation [13]. Based on a compilation of previous studies, Turner concluded that the strain rate and magnitude are related to the strain stimulus by the following equation [28].

$$
E=k \sum_{i=1}^{n} \varepsilon_{i} f_{i}
$$

where $E$ is the strain stimulus, $k$ is a constant of proportionality, $\varepsilon$ is the strain magnitude, and $f$ is the strain frequency. Equation (1.1) implies that a high magnitude, low frequency strain signal would produce the same anabolic response as a low magnitude high frequency strain signal.

Contemporary research over the last 35 years has led to five conclusions about bone adaptation and growth.

1) Bone adaptation is driven by dynamic, rather than static loading $[8,19]$.

2) The strain stimulus induced in bone is directly proportional to both magnitude and frequency of the strain signal [14, 28-30].

3) Only a short duration of mechanical loading is necessary to initiate an adaptive response due to fairly rapid cellular response saturation [19, 23, 29].

4) Sensitivity reestablishment is needed after saturation takes place in order to restore osteogenic capability $[16,17,19,23]$.

5) Bone cells adapt to customary loading over the long term making them less responsive to routine stimuli [23].

\subsection{Bone Sample}

The bone sample used for testing was a right porcine tibia. The tibia was chosen for the relatively flat surface it provides for bonding of the smart material. The tibia was harvested, cleared of soft tissue, and mounted in Polymethylmethacrylite (PMMA) by the Calhoun Research Lab affiliated with the University of Akron Department of Biomedical Engineering. The bone was mounted such that 1.25 inches of bone surface was exposed. 


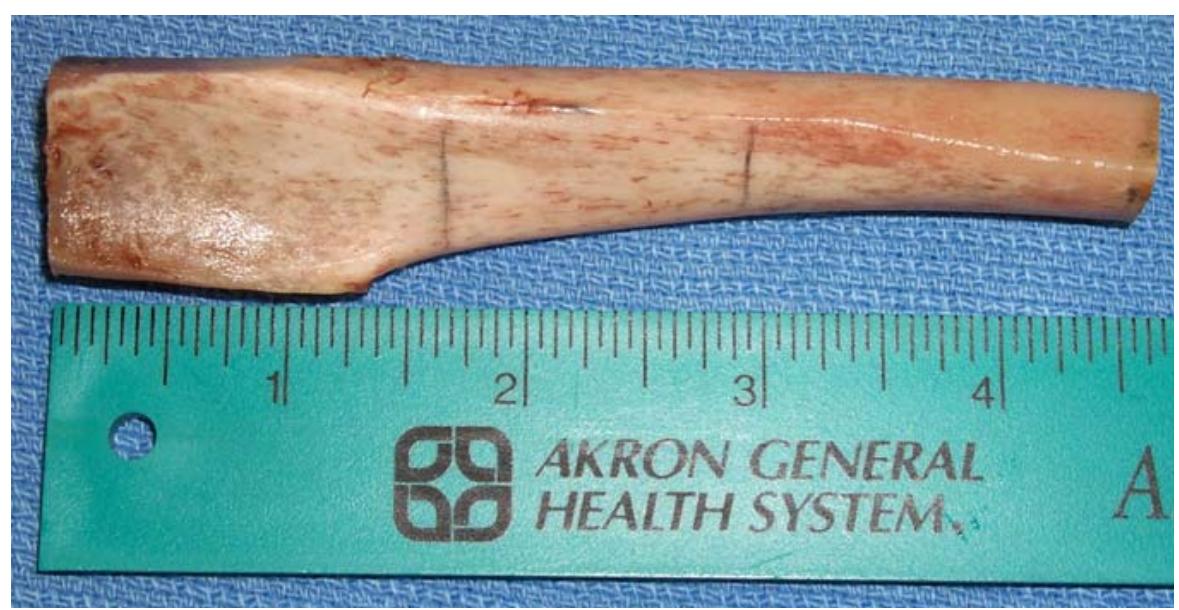

Figure 1.3: Total bone sample length (right porcine tibia)

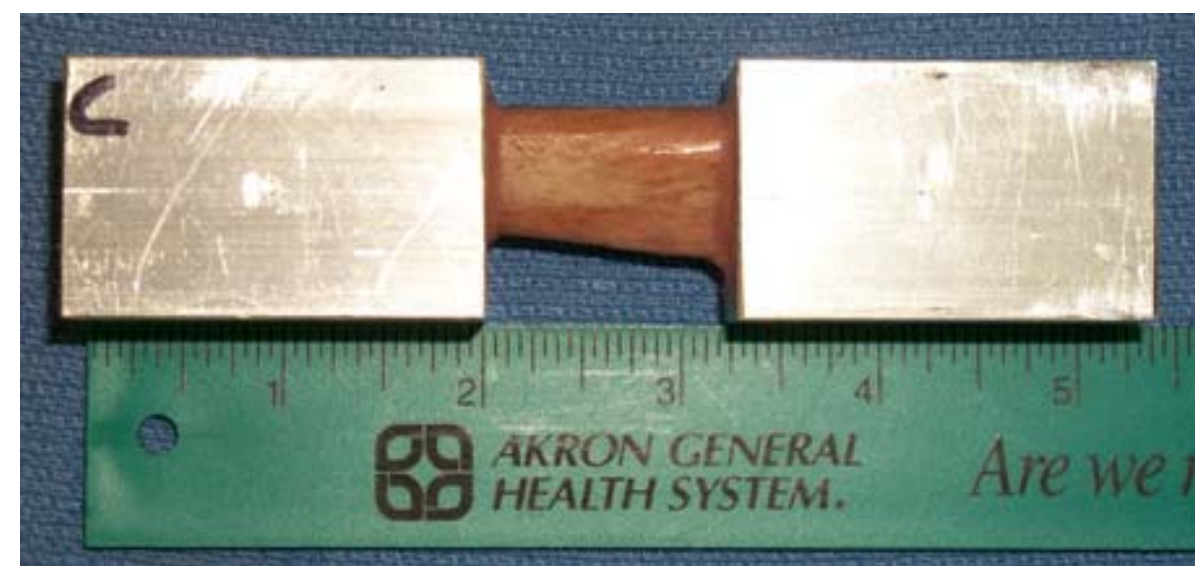

Figure 1.4: bone sample mounted in PMMA and aluminum blocks

\section{MAGNETISIM}

This paper is focused on the transduction of magnetic fields into mechanical strain in order to promote bone growth. Magnetic fields allow for the remote actuation of different smart materials and have a proven record of safety within the clinical setting. A background of magnetic fields and magnetism will be discussed to preface magnetic actuation of the smart materials under investigation.

\subsection{Magnetic Fields}

A magnetic field $H$ is generated by an electric charge passing through a conductive material or by the orbital spin of electrons within an atomic structure. The intensity of a 
magnetic field is measured in Amperes per meter (A/m) in SI units or Oersted (Oe) in CGS units. Magnetic fields create magnetic flux, which in a vacuum may be thought of as the spatial representation of a magnetic field. Figure 2.1 shows four different modes of magnetic field production and their associated magnetic flux paths.

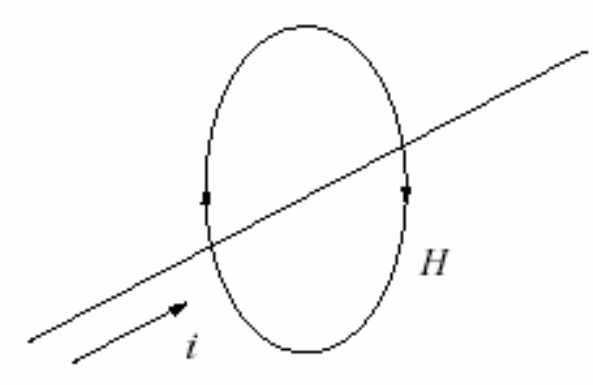

(a)

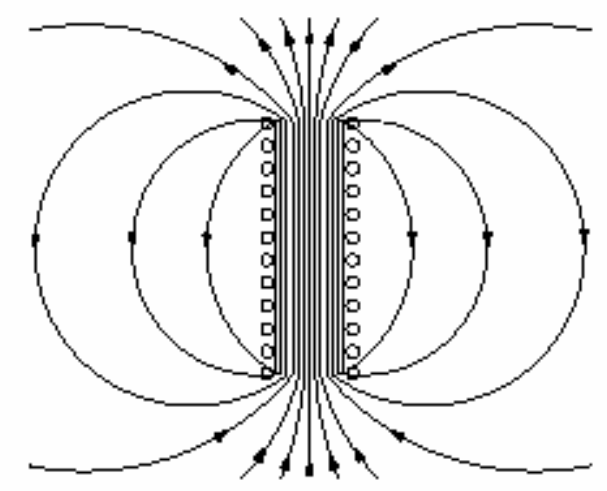

(c)

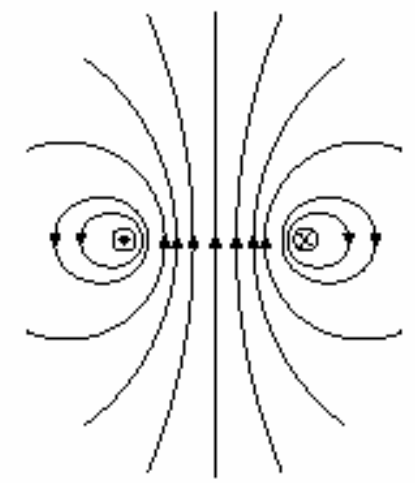

(b)

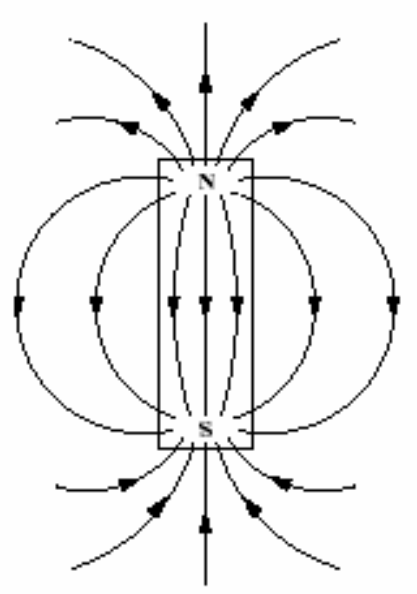

(d)

Figure 2.1: Magnetic fields created by (a) current through a conductive wire (b) current through a conductive loop (c) current through a series of conductive loops, or solenoid (d) permanent bar magnet [11]

When a magnetic field passes through a magnetically permeable medium, a magnetic induction $B$ occurs. Magnetic induction is also termed the flux density and is measured in Tesla (T) in SI units and Gauss (G) in CGS units. Magnetic field and magnetic induction are related by the permeability $\mu$ of the material residing in the flux path

$$
B=\mu H
$$


where $B$ is the magnetic induction, $H$ is the magnetic field strength, and $\mu$ is the magnetic permeability of the material subjected to the magnetic field. In air, the permeability of free space $\mu_{o}$ is used to related $B$ and $H$.

When a field is applied to a material with an intrinsic magnetic dipole, a ferrous alloy for example, the magnetization of the material also contributes to the induced field $B$. Equation (2.1) becomes

$$
B=\mu(H+M)
$$

where $M$ is the magnetization of the material $(\mathrm{A} / \mathrm{m})$ subjected to the applied magnetic field $H$.

When describing the permeability of a material it is useful to employ the relative permeability $\mu_{r}$.

$$
\mu_{r}=\frac{\mu}{\mu_{o}}
$$

The relationship between $B$ and $H$ is illustrated in the $B$ - $H$ curve or hysteresis loop shown in Figure 2.2. As a magnetic field is applied to a demagnetized material $(B=0, H=0)$ the $\mathrm{B}-\mathrm{H}$ trace moves from the origin to the point corresponding to complete magnetization of the material. As the magnetic field is decreased from this point back to zero, the demagnetization of the material occurs at a distinct rate from the initial magnetization. When the field again reaches zero $(H=0)$ a residual amount of magnetization remains in the material $(B \neq 0)$. The phenomenon that leads to two different states at $B=0$ is known as the magnetic hysteresis. 


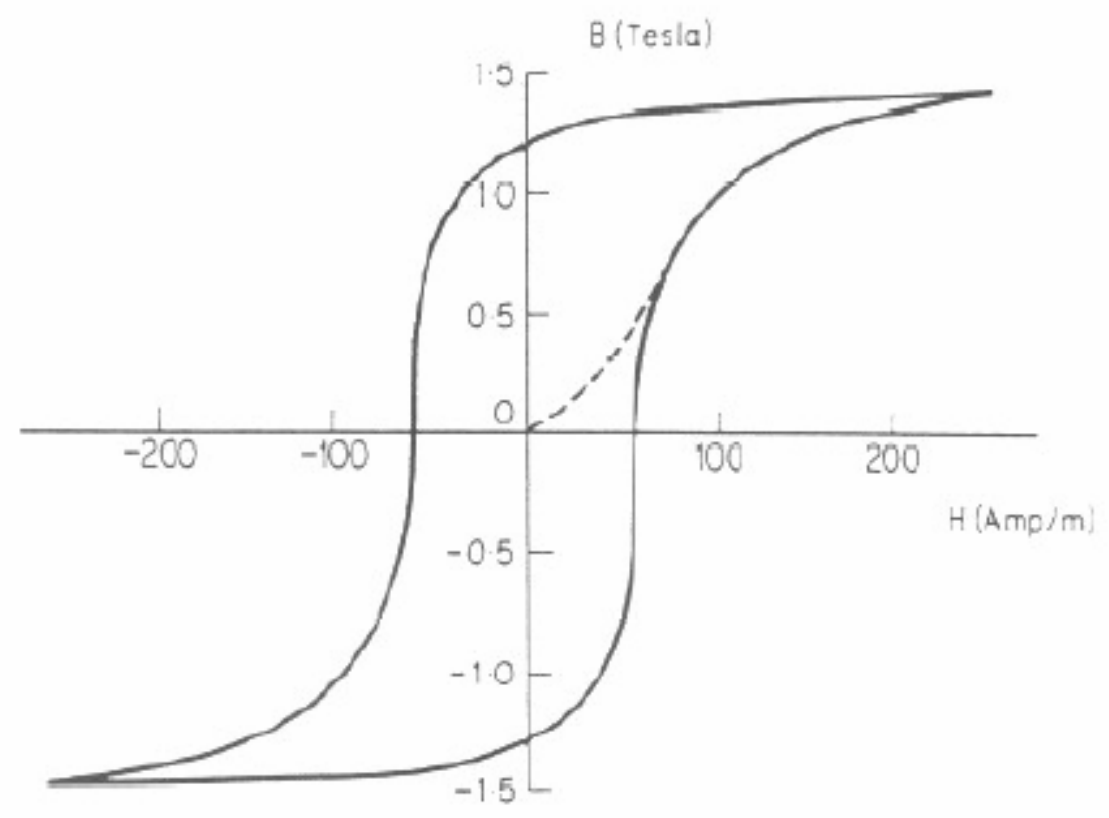

Figure 2.2: $B-H$ Curve for a typical ferromagnetic material

\subsection{Magnetic Field Measurements}

To quantify the level of field and induction present in the experiments conducted in this study, it is useful to develop an equation relating the applied voltage to a drive coil and the resulting magnetic field intensity. Beginning with Ampere's Law, the magnetic field $H$ in a coil is described by

$$
\oint H \bullet d l=N I
$$

where $d l$ is the differential length of the coil, $N$ is the number of turns, and $I$ is the current through the coil. Equation (2.3) may then be simplified to

$$
H=\frac{N I}{L} \text {. }
$$

Magnetic induction, on the other hand, is derived from the differential form of Faraday's law states that a change in magnetic flux $\frac{d \phi}{d t}$ is proportional to an electro motive force (e.m.f.) induced in a conductor. Coupled with Lenz's law which states that the induced e.m.f. occurs in the opposite direction of the applied magnetic field, the relationship between induced voltage and flux becomes 


$$
V=-N \frac{d \phi}{d t}
$$

Since magnetic induction is defined as flux per unit area

$$
B=\frac{\phi}{A}
$$

Equations (2.5) and (2.6) may be combined to relate magnetic induction and induced e.m.f.

$$
V=-N A \frac{d B}{d t}
$$

and then rearranged to easily determine the induced field by integrating the induced voltage

$$
B=\frac{-1}{N A} \int V d t
$$

In practice, the current applied to the drive coil was measured as well as the voltage induced in a two turn "pickup" or sensing coil on the inside of the drive coil. Measurement of the applied current provided the level of applied field though Equation (2.4) and measurement of the induced voltage provided the induction through Equation (2.8). It is noted that due to the large air gap and relatively large reluctance of the Terfenol-D composite, the measured magnetic induction versus field curve is a straight line that corresponds to that of air.

\subsection{Characteristics of Magnetic Materials}

As discussed previously, the permeability of a material quantifies how the material responds to an applied magnetic field. As a result, metals may be broken down into three classes based on the relative permeability $\mu_{r}$ of the material. Dimagnetic and paramagnetic materials have relative permeabilities close to one while ferromagnetic materials have permeabilities greater than one. Terfenol-D has a relative permeability between four and five while iron has a relative permeability of up to 200,000 .

On a microscopic level, domains residing in the crystalline structure carry a net magnetic dipole. As a ferromagnetic material is subjected to an increasing magnetic field, favorably-oriented domains will grow in size at the expense of unfavorably-oriented domains. The peak in the $B-H$ curve corresponds to the microscopic condition when all of the dipoles are aligned parallel to the applied magnetic field. Figure 2.3 gives a schematic representation of how domain structure changes as a function of applied magnetic field. 

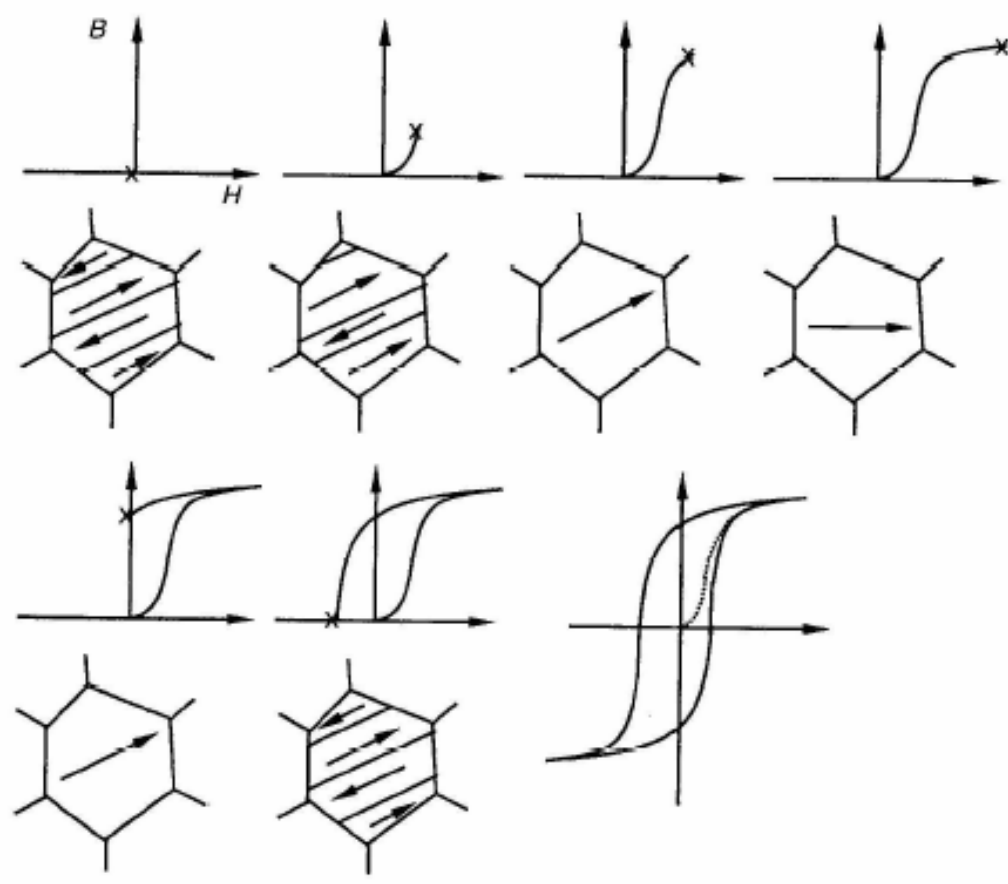

Figure 2.3: Domain structure as a function of applied magnetic field [13].

\section{3. "SMART" MATERIALS}

"Smart" materials are a class of materials that have the ability to significantly change physical characteristics such as shape or stiffness with external stimuli. Two such materials have been chosen for investigation in this paper, shape memory alloys (SMAs) and magnetostrictors. The shape memory effect and the process of magnetostriction will now be described along with methods to effect their remote activation through magnetic fields.

\subsection{Shape Memory Alloys}

Shape memory alloys (SMAs) are an attractive method to load bone at low frequencies because of the large strain possible as a result of the shape memory effect. The shape memory effect may be described as the ability of an alloy to transform from one state to a second state and then passively transform back to the original state. The ability to "remember" the original state lies in the transformation between two crystalline structures, the martensite phase and the austenite phase. The martensite phase is the low temperature 
phase and is highly ductile. The austenite phase exists at high temperatures and is mechanically stiffer than the martensite phase.

Figure 3.1 shows the cycle for transforming the alloy between the two phases. In a cooled state, the alloy exhibits a martensitic structure. As the alloy is heated, the transition to the austenite phase begins at the austenite start $\left(A_{s}\right)$ temperature and ends at the austenite finish $\left(\mathrm{A}_{\mathrm{f}}\right)$ temperature. Similarly, as the alloy is allowed to cool, the transition to the martensite phase begins at the martensite start $\left(\mathrm{M}_{\mathrm{s}}\right)$ temperature and ends at the martensite finish $\left(\mathrm{M}_{\mathrm{f}}\right)$ temperature.

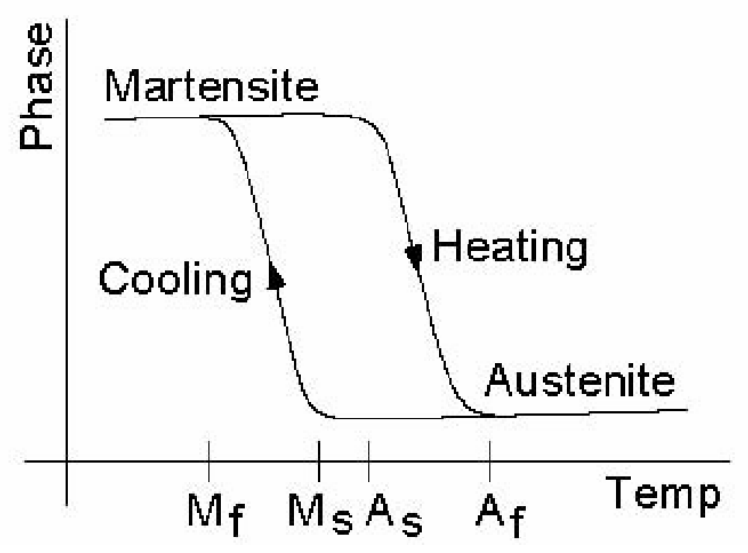

Figure 3.1: Phase transition curve for shape memory alloys

To mechanically load bone, a SMA could be heat set in the austenite phase to produce a rigid shape, a rod with a curvature for instance. Once cooled, the rod would easily deform back to a straight rod. This pliable straight rod could be applied to a long bone and then remotely heated to produce a shape change, the shape memory effect. As the body dissipates the heat, the alloy would return to the native straight pliable phase. To accomplish this, an alloy would necessitate a narrow hysteresis profile similar to Figure 3.1 with an $\mathrm{M}_{\mathrm{f}}$ temperature slightly above normal body temperature. One such alloy is Nitinol, an alloy with approximately equal parts nickel and titanium originally developed by the Naval Ordinance Lab.

For the purpose of loading bone, the SMA requires a remote method to develop heat and allow the shape transformation to occur. High frequency electro-magnetic fields that develop eddy currents within the alloy may create sufficient heat to promote the phase change from martensite to austenite. Several studies have shown the effectiveness of using a 
"pancake" type coil in the radio frequency range to promote the shape memory transition in animals in-vivo $[22,24]$.

Although Nitinol is a proven biocompatible material and has been shown to produce phase transformations with remote inductive heating, its slow response time severely complicates its implementation in clinical treatment.

\subsection{Magnetostrictors}

Magnetostriction is the process by which a material undergoes a shape change in response to a change in magnetization. The process may be graphically described by considering Figure 3.2. Above the Curie temperature, magnetostrictive materials are paramagnetic and do not exhibit magnetic dipoles. Cooling of the material through the Curie temperature, however, leads to a domain structure and corresponding spontaneous magnetostriction. As a magnetic field is applied to the ferromagnetic material, the domains rotate and align with the magnetic field, thus causing the material to deform.

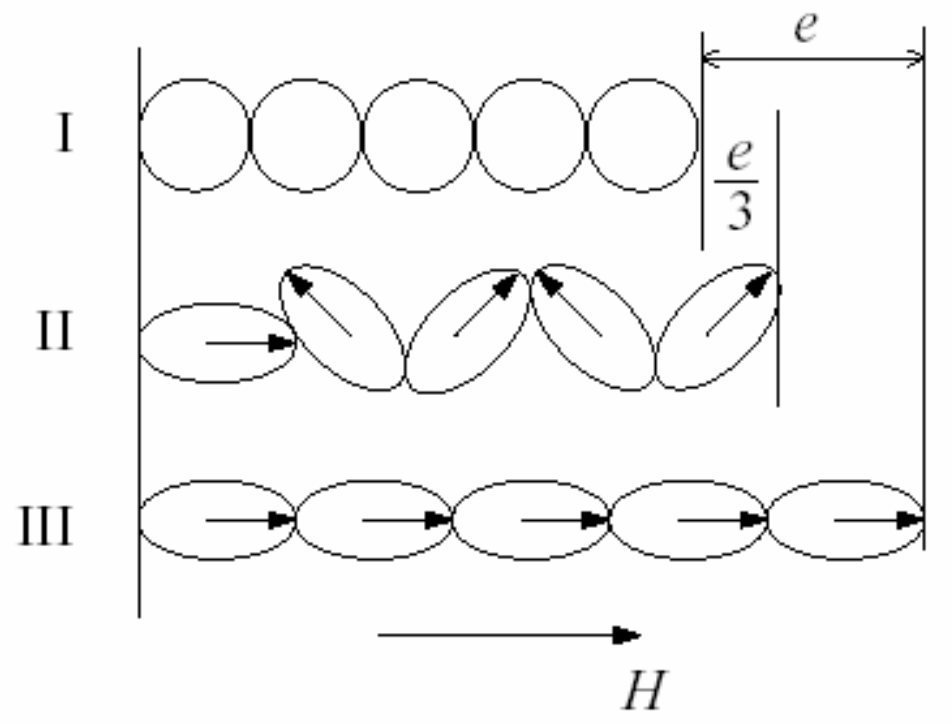

Figure 3.2: Process of magnetostriction I) above Curie temperature II) below Curie temperature with no applied field and III) below Curie temperature with applied field.

Even though all ferromagnetic materials exhibit at least a small amount of magnetostriction caused by domain wall motion, some materials such as rare earth metals are capable of "giant" magnetostriction in excess of $1000 \mathrm{ppm}$. The most advanced gain 
magnetostrictive material, Terfenol-D, is an alloy of terbium (TER), iron (FE) and dysprosium (D), first developed at the Naval Ordinance Lab (NOL).

One important characteristic of Terfenol-D is the need to apply a preload to the material in order to maximize the strain and force potential. In Figure 3.3, the application of a compressive preload aligns the domains in the direction perpendicular to the preload.

When a field is applied, maximum magnetostriction is achieved through 90-degree rotation of the magnetic domains. It is noted that positive and negative fields produce opposite magnetizations but the same magnetostriction.

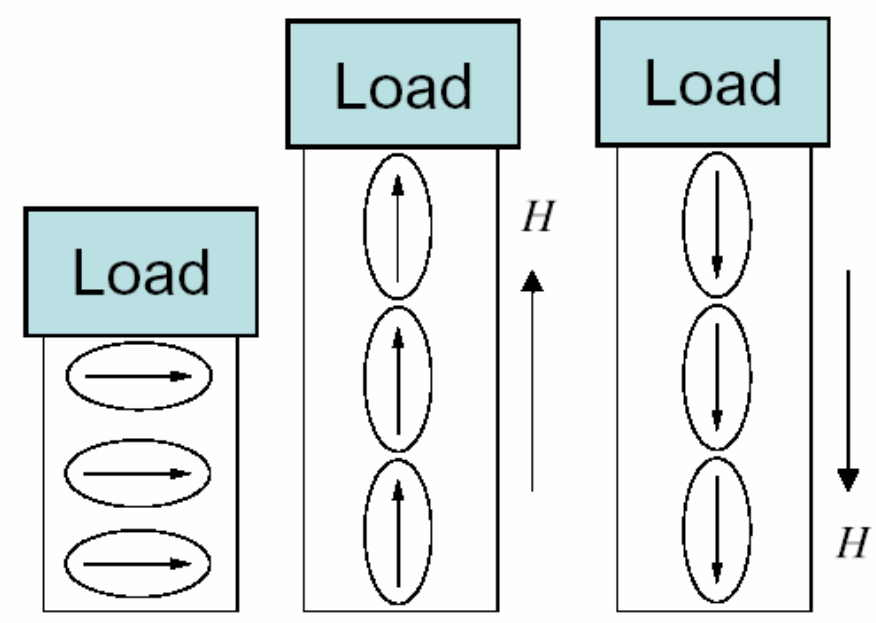

Figure 3.4: Effect of preload on Terfenol-D

\section{FABRICATION}

This section describes the methods and techniques used to fabricate and actuate the bone loading device. The Terfenol-D composite fabrication method is derived from the work of A.P. Mortensen on the characterization, modeling, and dynamic implementation of Terfenol-D composites [10]. All components were fabricated in the Smart Materials and Structures Lab at The Ohio State University Department of Mechanical Engineering.

\subsection{Terfenol-D Composite}

Terfenol-D in monolithic form exhibits considerably higher saturation magnetostriction than in composite form; however, the composite form increases the 
mechanically flexibility of the alloy for bone loading applications while enabling biocompatibility through the use of an appropriate epoxy matrix. A soft epoxy resin with a low modulus of elasticity was used as a binder to minimize magnetostriction loss. Derakane 411-C-50 epoxy vinyl ester resin was used as the binder. Methyl Ethyl Ketone Peroxide (MEKP, Methylethylketonehydroperoxide) was the catalyst for the epoxidation reaction and Cobalt Napthenate (CoNap) was the reaction promoter. The resin was mixed in a $97.8 \%$ epoxy, 2.0\% MEKP, and $0.2 \%$ CoNap ratio by mass. A step-by-step procedure for mixing and casting the composite is included in Appendix A.

The Terfenol-D particles were manufactured by a ball-milling process producing low aspect ratio particles ranging in size from 106-300 microns. The composite was mixed in an open air container with the appropriate amount of Terfenol-D particulate to achieve an approximately $50 \%$ volume fraction between the resin and the Terfenol-D. While casting the sample in a vacuum would have aided in the elimination of air pockets within the matrix, it has been shown that the performance of the vacuum-cast composite is comparable to that achievable through open air casting [4]. The mixed resin/Terfenol-D particles were then cast in the aluminum mold sealed with silicon. A nickel plated neodymium iron boron (NdFeB) magnet was quickly slid over the mold to align the particles during the composite cure. To ensure a complete cure, the composite and mold were placed in a convection oven at $70^{\circ} \mathrm{C}$ for 6 hours. Following the cure the composite was machined to achieve squared ends. Two samples were mixed and cast, one with a $50.2 \%$ and the second with $42.0 \%$ volume fraction of Terfenol-D. The $50.2 \%$ composite sample was cut into two approximately one inch length rods. One piece was used for scanning electron microscopy investigation and the other piece was cut in half along the long axis to create a semicylindrical geometry able to be bonded to the bone sample. 


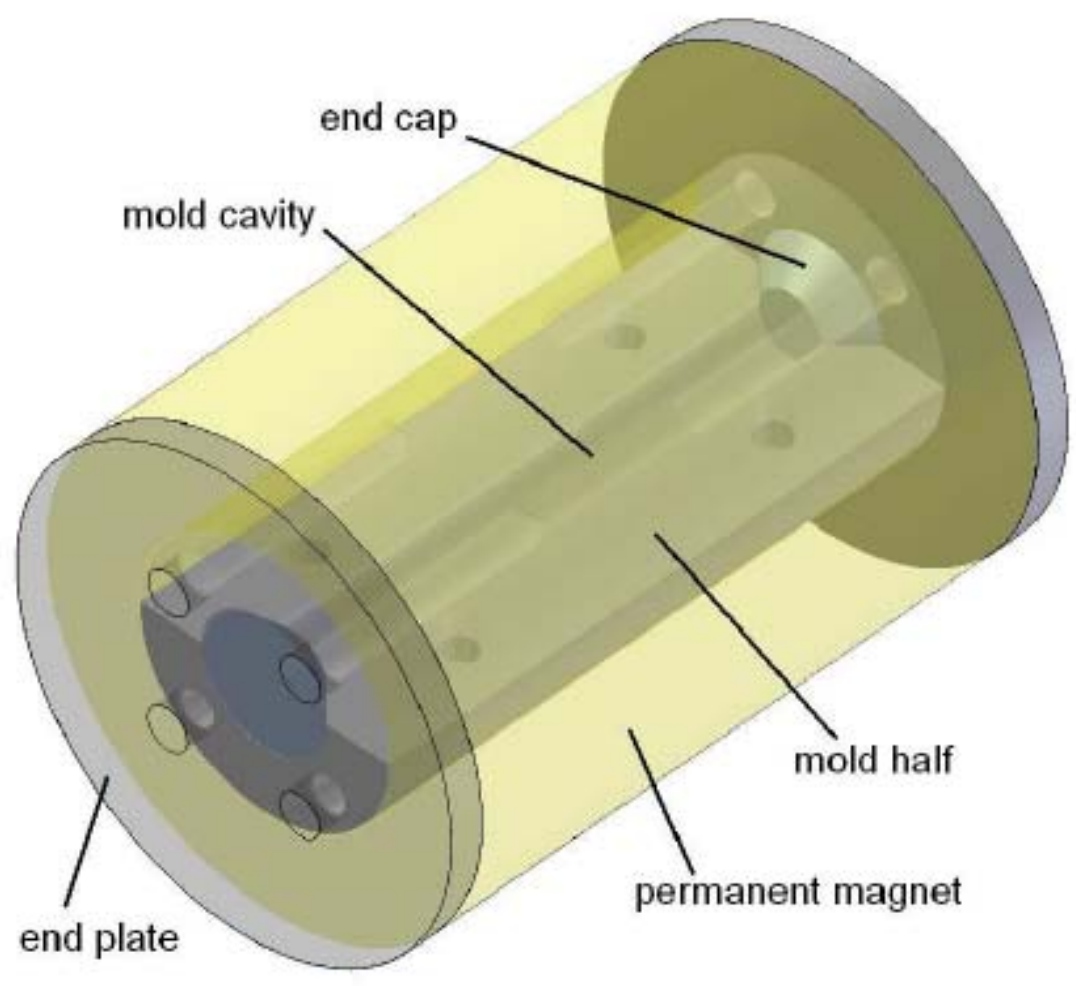

Figure 4.1: Casting mold with permanent magnet in position [11]

\subsection{Drive Coil}

A drive coil, or solenoid, able to produce sufficient field to activate the composite was necessary. The need to keep the core of the solenoid large enough to fit the bone sample presented a challenge. An efficient activation coil minimizes the amount of flux that passes through the air gaps between the composite and coil. However, since the crosssectional area of the bone sample is much larger than that of the composite and the composite reluctance is high, much of the flux will leak away from the composite. A thick coil was wound in order to produce enough flux to sufficiently activate the composite.

The drive coil was constructed out of 20AWG magnet wire. A spindle was machined out of steel and aluminum and was attached to a variable speed motor in order to control the layering of the wire. A thin layer of heat resistant epoxy was spread between each layer of coil with a rubber spatula to maintain structural integrity of the coil. A two layer pick-up coil was placed inside the drive coil to measure inductance. The drive coil has a resistance of $18.9 \Omega$ and a field rating of $1.6 \mathrm{kA} / \mathrm{m} / \mathrm{V}$. 


\subsection{Compression Bracket}

During cure of the composite, a residual precompression of the Terfenol-D particles is established. The behavior of the composite under load, including precompression and external loading was characterized. To that end, a compression bracket was constructed that allows to measure the blocked force generated by the sample under varied magnetic fields and to investigate the effects of various external loads. The bracket blocks were machined out of aluminum and were fastened together with 2 brass screws in order to keep the assembly non-magnetic. A FlexiForce ${ }^{\circledR}$ sensor from Tekscan, Inc. of South Boston, MA was slid between an aluminum block and the composite in order to measure and acquire axial forces.

If it was experimentally found that the composite performance optimum occurred under a given external load, the composite would need to be applied to the bone in a precompressed state equivalent to the external load. For this reason, the bottom of the bracket and the bottom of the composite are flush. 


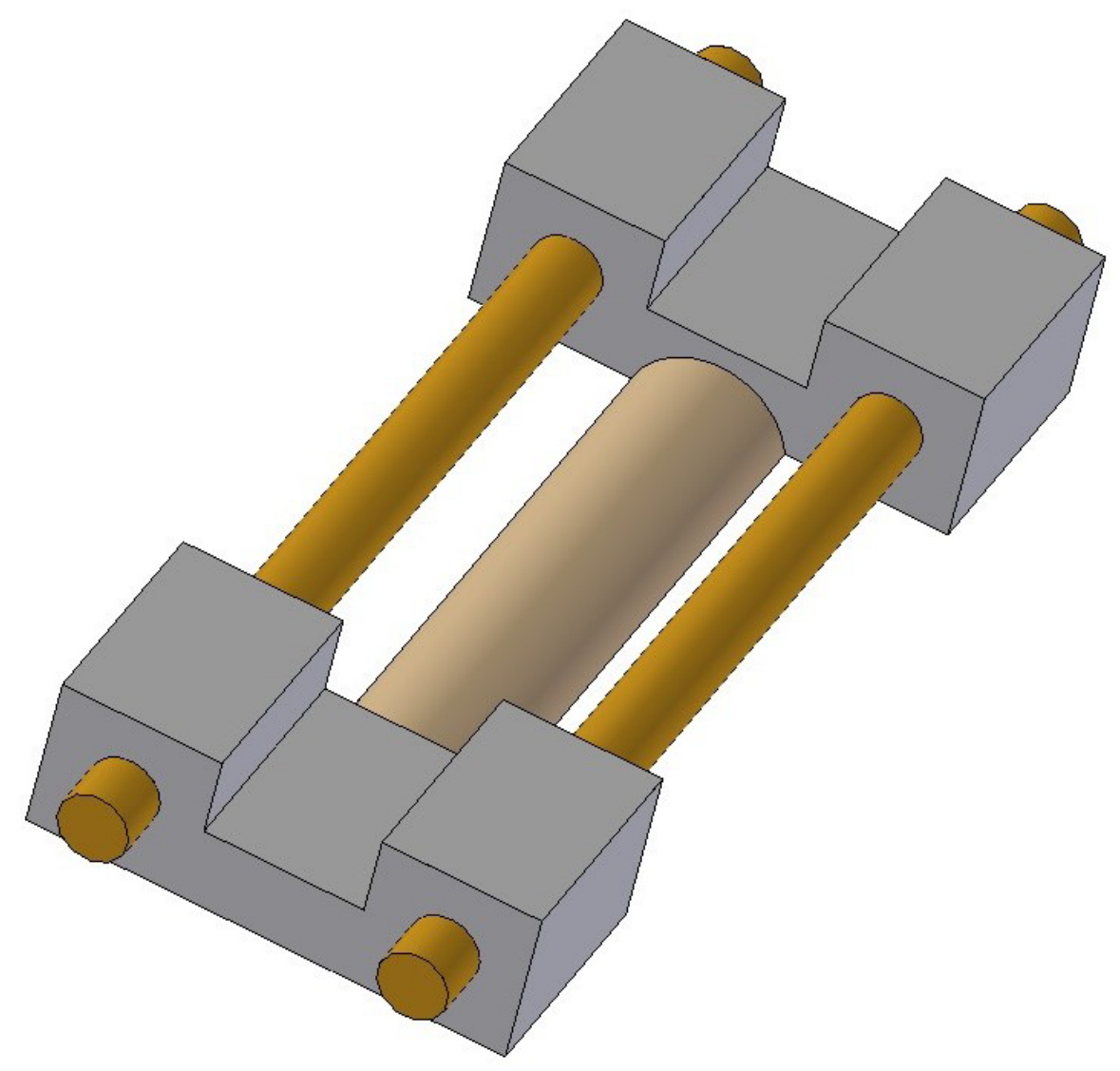

Figure 4.2: Compression bracket with Terfenol-D composite in position

\section{CHARACTERIZATION OF TERFENOL-D COMPOSITE}

To further understand the behavior of Terfenol-D composites, different methods of characterization where conducted. A linear model of the composite/adhesive/bone system was developed to describe the bone strain developed as a function of the applied magnetic field. Microscopic image analysis attempted to quantify the crystalline orientation achieved under the current fabrication methods. Load analysis was conducted in order to determine the stress levels that maximize the performance of the composite. 


\subsection{Linear Modeling}

A continuum approach to modeling the active system was used and is shown in

Figure 5.1. The composite is at the top and the bone is at the bottom, with an adhesive layer between the two. Since the cyanoacrylate (superglue) that was used to bond the composite to the bone is viscoelastic, a shear loss will result between the Terfenol-D and the bone.

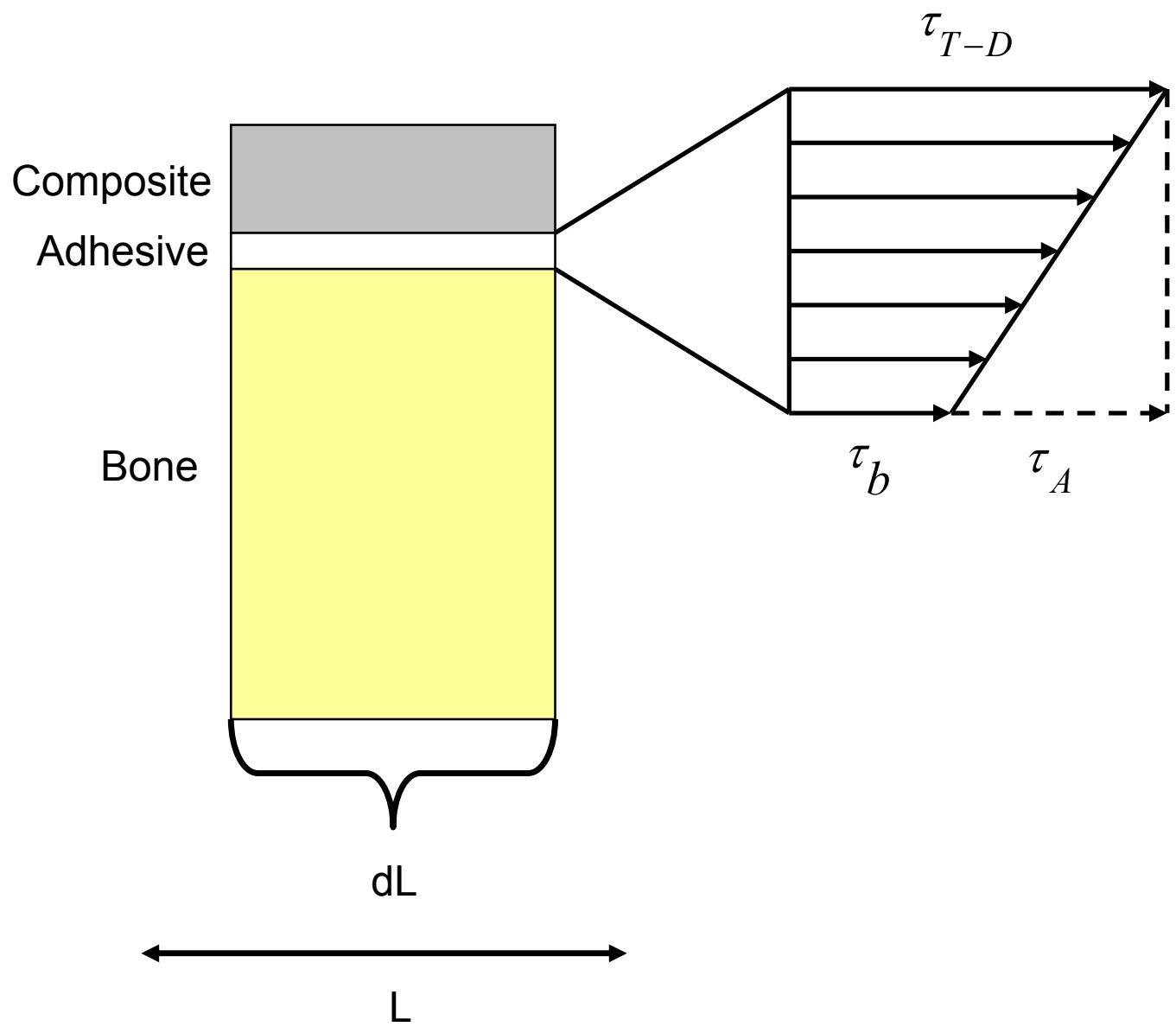

Figure 5.1: Differential continuum model of the composite/adhesive/bone system

Linear modeling of the composite begins with the constitutive piezomagnetic relation for deformation

$$
\varepsilon=\frac{\sigma^{H}}{E^{M}}+q H
$$

where $\varepsilon$ is the strain, $\sigma$ is the axial stress induced by the applied magnetic field $H, E^{M}$ is the Young's modulus at constant magnetic field, and $q$ is the magnetoelastic coupling coefficient. For this paper, a $q$ value of $2.71 \times 10^{-6} \mathrm{~m} / \mathrm{A}$ will be used [11]. Equation (5.1) 
describes the strain on the bone surface. This strain value may then be transformed into the shear stress developed on the composite/adhesive interface by

$$
\tau_{T-D}=\frac{E^{M} \varepsilon A_{c}}{A_{T-D / A d h e s i v e}}
$$

where $\tau_{T-D}$ is the shear stress developed by the composite, $A_{c}$ is the cross sectional area of the composite, and $A_{T-D / \text { Adhesive }}$ is area of the composite/adhesive interface.

Once the performance of the composite has been quantified, the action of the adhesive layer may be described. The adhesive layer was modeled as a spring and damper system in parallel. The result is a complex shear modulus with the form

$$
G_{A}=k+i b(\omega)
$$

where $G_{A}$ is the complex shear modulus of the adhesive, $k$ is the real component of the modulus, and $b$ is damping coefficient. In addition to Equation (5.3), the complex shear modulus may be described as a ratio of $G_{\text {diss }}$ to $G_{\text {store }}$ representing the dissipated (imaginary) and storage (real) moduli respectively. This ratio is also referred to as the "loss" factor.

$$
G_{A}=\frac{G_{\text {diss }}}{G_{\text {store }}}
$$

Once the shear through the adhesive is modeled, the strain and applied force applied to the bone may be described.

$$
\begin{gathered}
F_{b}=\tau_{b} w \int d l \\
\varepsilon_{b}=\frac{F_{b}}{E_{b} A_{b}}
\end{gathered}
$$

where $F_{b}$ is the force exerted by the bone on the deforming composite, $\tau_{b}$ is the shear stress developed on the bone surface, $w$ is the width of the adhesive/bone interface, $d l$ is the differential length along the adhesive/bone interface, $\varepsilon_{b}$ is the strain developed on the bone surface, $E_{b}$ is the modulus of elasticity for the bone, and $A_{b}$ is the cross sectional area of the bone. 


\subsection{Microscopic Image Analysis}

During the casting of the composite, the Terfenol-D particles have the opportunity, however brief, to settle towards the bottom of the mold. In addition, the permanent magnet used to align the particles during the cure was designed to produce a field of $140 \mathrm{kA} / \mathrm{m}$ in order to preferentially orient the particles [11]. Microscopic image analysis was used to investigate and quantify the distribution and crystalline orientation of the particles within the cured composite. A brief discussion about how a scanning electron microscope operates and how to interpret the image results will be given followed by the results of the Terfenol-D composite analysis.

\subsubsection{Scanning Electron Microscopy}

Scanning Electron Microscopy (SEM) begins with the formation of an electron beam in a vacuum. The electrons are emitted from a filament located approximately one meter from the sample (cathode) and travel towards the sample mounted in conductive Bakelite $\mathbb{R}$ (anode.) The electron beam passes through a series of electrically activated coils, which focus the beam and align it with the sample. The electrons emanating from the filament are termed high energy primary electrons. Once the primary electrons hit the sample, two phenomena occur. First, a portion of the high energy primary electrons are scattered by the structured atomic nuclei in the sample. Second, a portion of the atoms close to the surface of the sample are energized by the primary electrons and emit lower energy secondary electrons. Scattered primary and secondary electrons are then captured by detectors located close to the sample surface.

Two types of detectors are frequently used with SEMs to detect both types of scattered electron. The first type of detector is called an Everhart-Thornley Detector (ETD). The ETD detects lower energy, secondary electrons emitted by the sample. Since these electrons originate close to the sample surface, the ETD provides a topographical image. Highly angled surfaces appear brighter than the sample surface using an ETD. The second type of detector is a Back-Scatter Detector (BSD), which picks up scattered primary electrons. The amount of scatter is a function of the atomic mass of the sample with larger nuclei scattering proportionately more electrons. As a result, brighter areas on the BSD images represent, on average, higher atomic mass while darker areas, on average, represent 
lower atomic mass. Often the EDT and BSD detectors are used simultaneously to produce an image that describes both the topography and atomic content of the material.

In addition to the high resolution images obtained with the EDT and BSD detectors, the SEM is also capable of producing an Orientation Image Map (OIM) of a sample. The OIM can map the surface of a sample along grain boundaries. The detected electron diffraction pattern is compared against a database containing many different crystalline structures. The resulting image yields regions with different colors corresponding to particular grain orientation.

\subsubsection{SEM Results}

Two composite samples were mounted in conductive Bakelite ${ }^{\circledR}$ by the Materials Science and Engineering Department at The Ohio State University as shown in Figure 5.2. The first sample contains three cross sectional discs from the end of the composite. These will be referred to as the radial cross sections. The exposed surface was then polished on a vibratory polisher for 48 hours. The second sample was cut from a one inch long semicylindrical mid-section of the same composite. This sample will be referred to as the axial cross section. The two samples give two different image surfaces at a perpendicular angle.
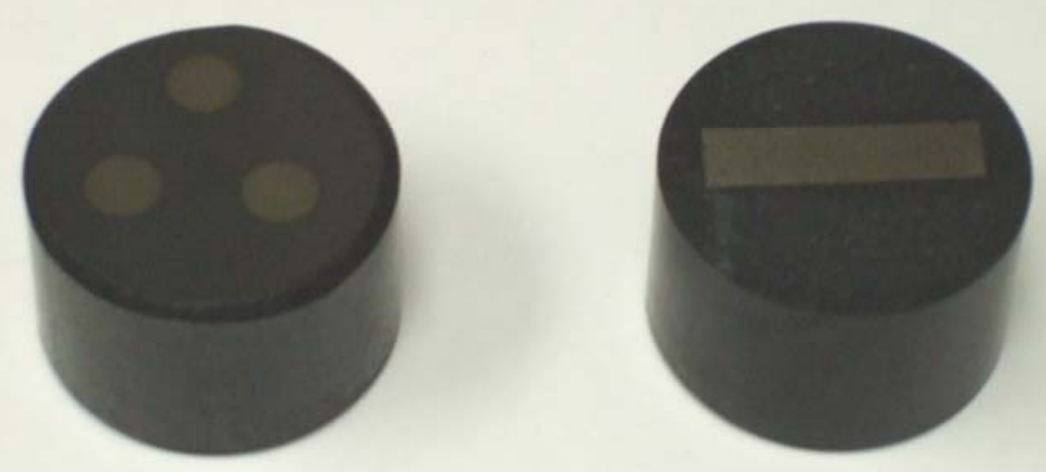

Figure 5.2: Radial cross sections (left) and axial cross section (right) mounted in conductive Bakelite

Figure 5.3 shows a radial cross section of Terfenol-D composite using an ETD and Figure 5.4 shows the same radial cross section with a BSD. The steep (bright) rings formed in Figure 5.3 correspond to the surface depressions in the epoxy as a result of the vibratory polishing. Figure 5.4 gives insight into the atomic composition of the surface. The epoxy 
matrix is represented by the darkest areas, followed by the gray iron rich areas, and finally the bright terbium/dysprosium rich areas.

Despite the advantages that an OIM of the sample surface might reveal, a reliable OIM analysis was not possible for two reasons. First, a very smooth surface is required to scatter the necessary amount of atoms into the detector to determine the crystalline structure. Only a few areas on the sample gave positive crystalline structures. Second, the crystalline structures that were determined did not index to any structure in the computer database.

The axial cross section shown in Figure 5.5 clearly shows that the particle orientation is not aligned with the magnetic field applied during cure, however the grain structure most likely is aligned with the magnetic field because of confirmed magnetostriction.

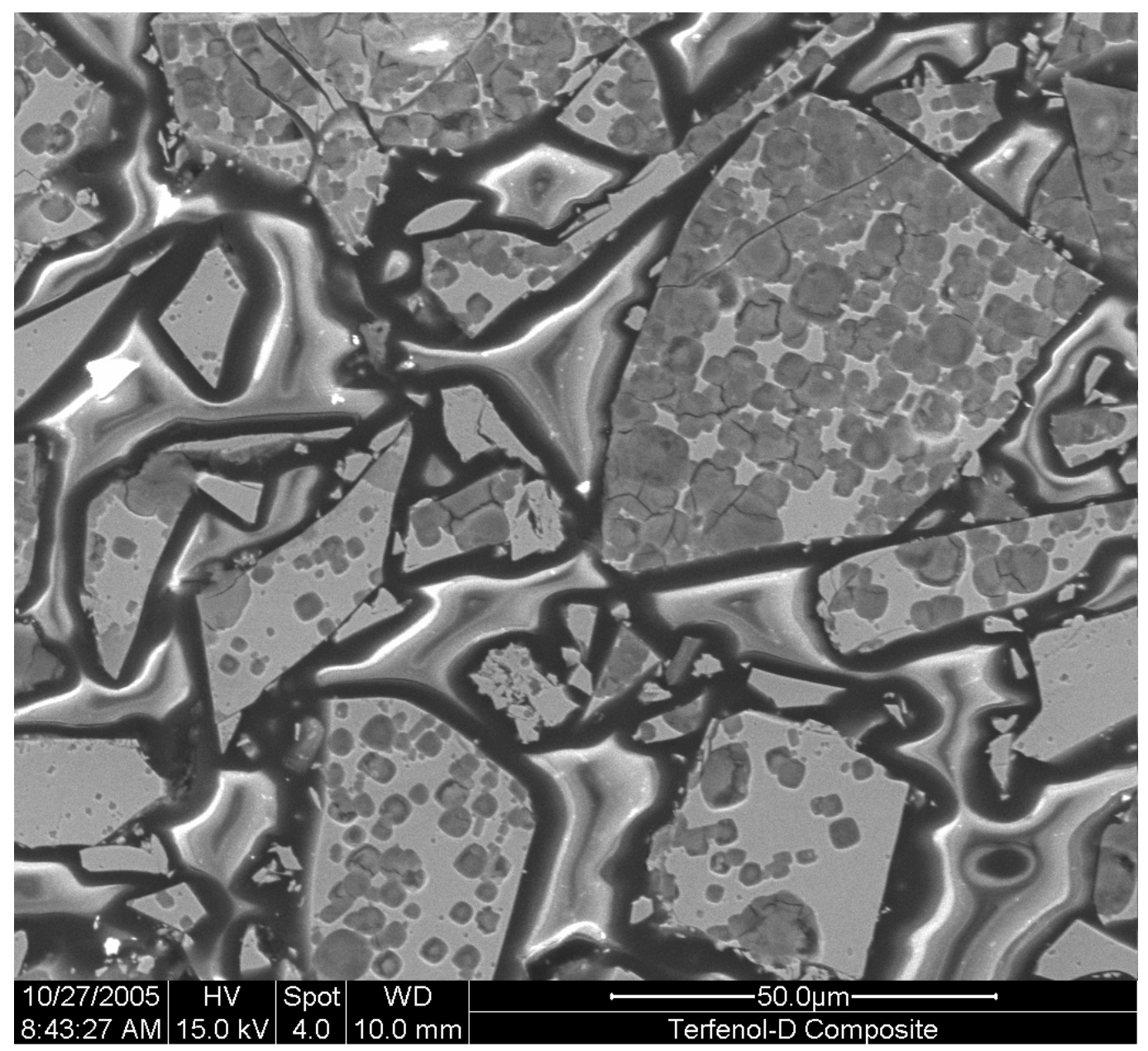

Figure 5.3: Radial cross section with Everhart-Thornley Detector 


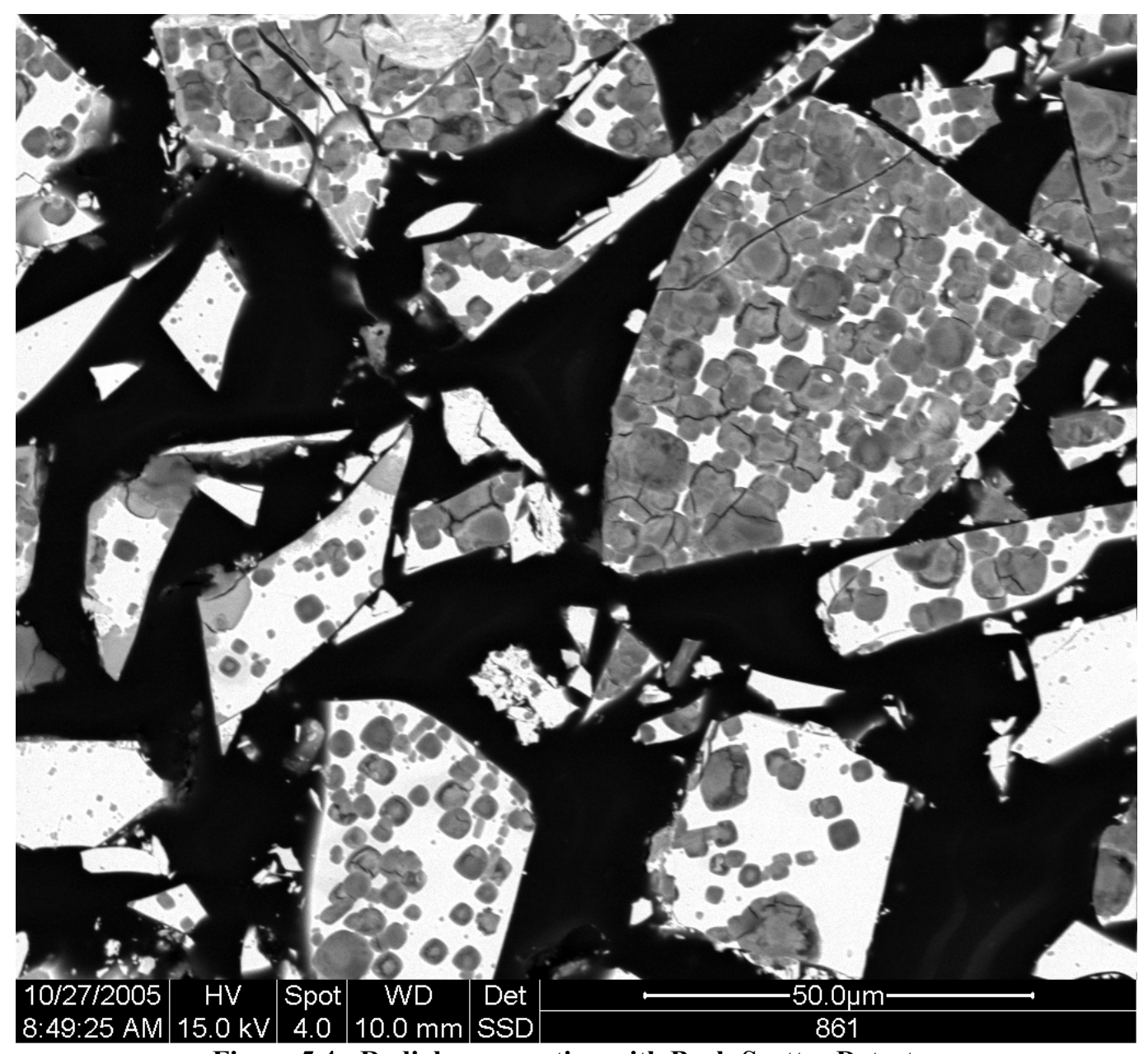

Figure 5.4: Radial cross section with Back-Scatter Detector 


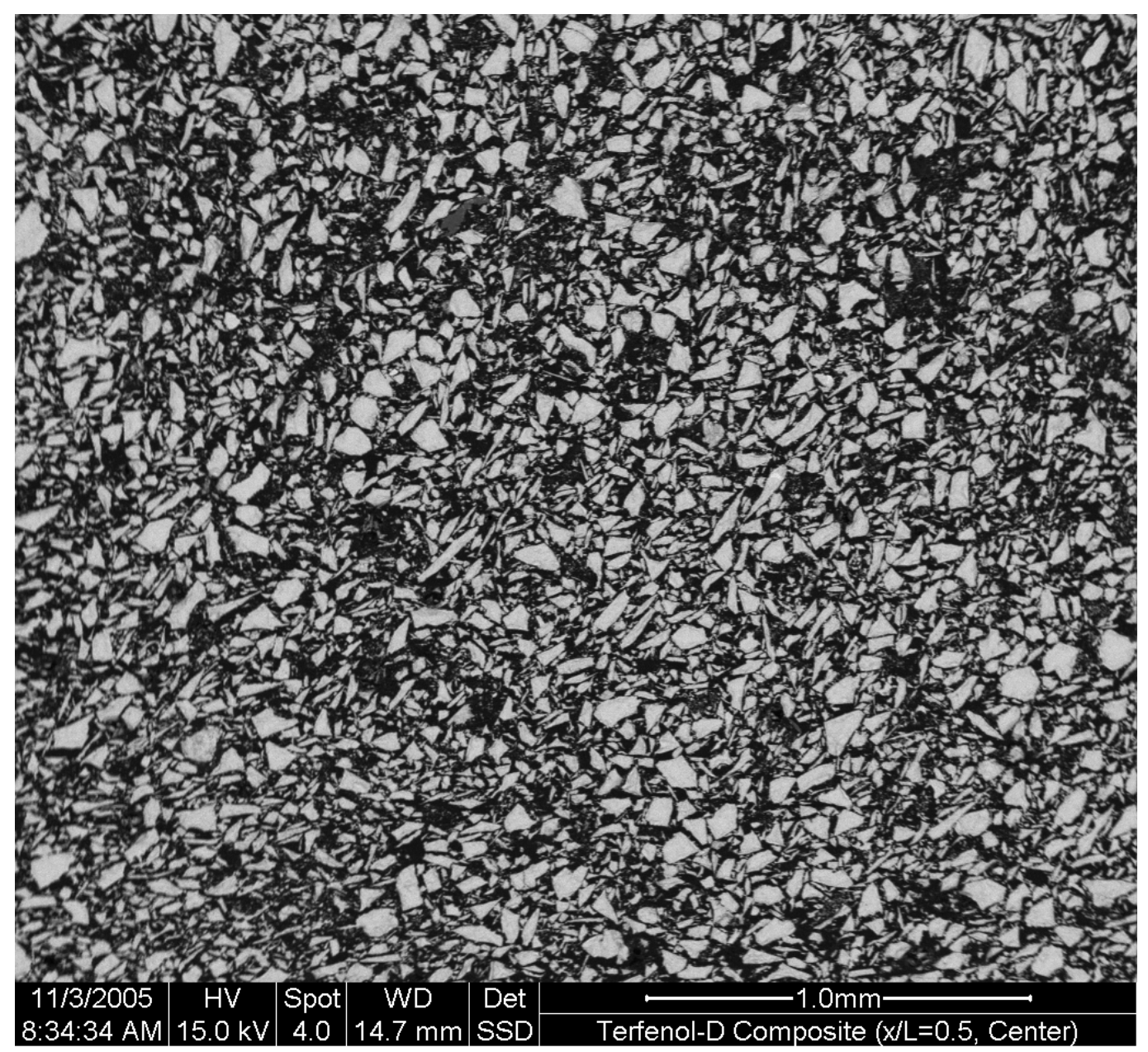

Figure 5.5: Axial cross section of sample (long axis of composite horizontal) with Back-Scatter Dectector

\subsection{Pre-stress Analysis}

A pre-stress on the composite is necessary in order to achieve maximum

magnetostriction. As the epoxy matrix cures, it will also contract inducing a certain amount of precompression on the Terfenol-D particles. It is not known, however, whether additional precompression is necessary. In order to answer this question, the composite was mounted in the compression bracket with a Flexiforce sensor and loaded with varying precomporession levels and then magnetically actuated. Appendix B gives a detailed setup for the Flexiforce sensor.

Figure 5.6 shows the blocked force production in the composite as the preload is increased from 0 to $35 \mathrm{lbf}$. Due to the relatively low resolution of the sensor, however, a discernable trend is not observed 


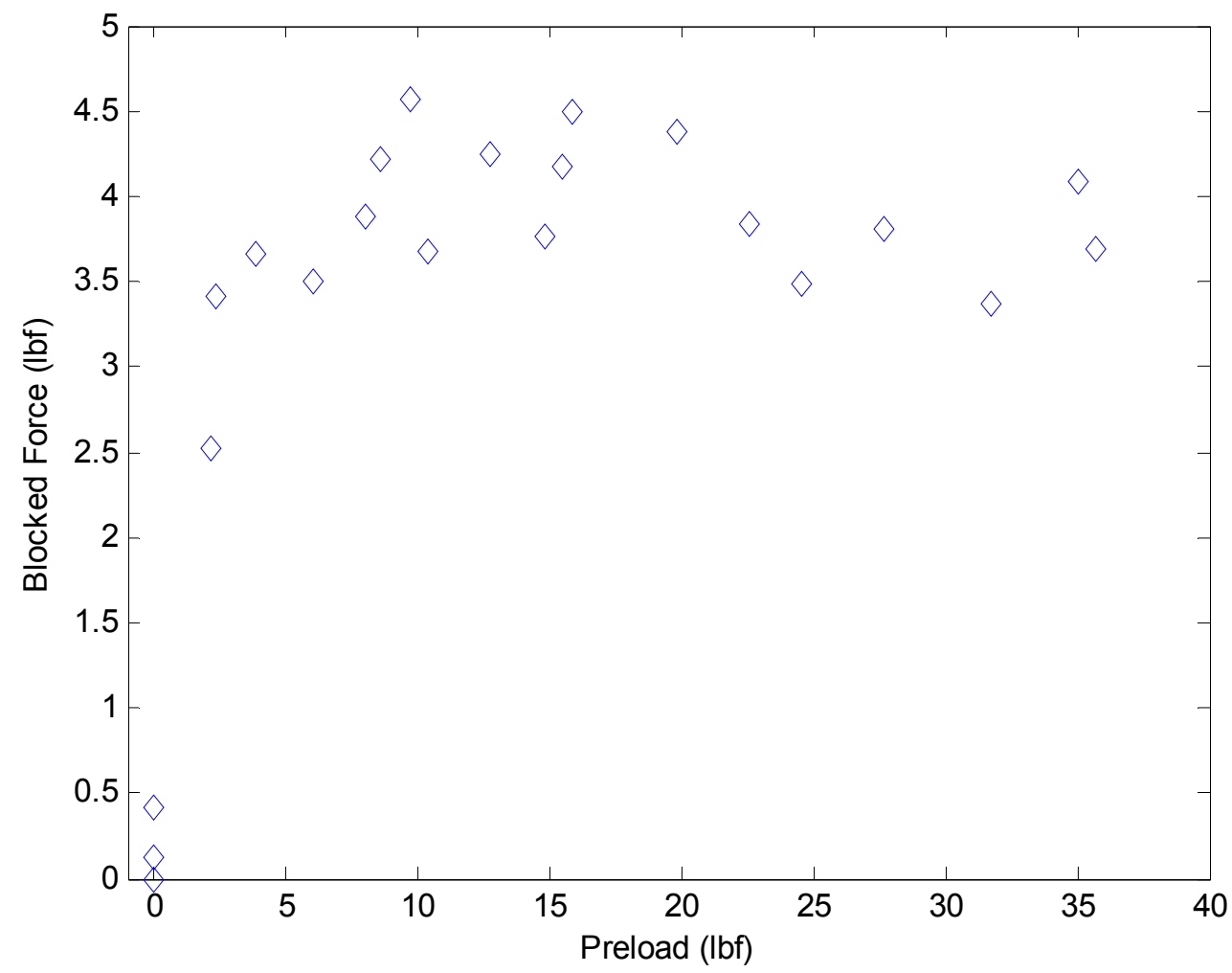

Figure 5.6: Preload effect on blocked force production in Terfenol-D composite 


\section{DYNAMIC BONE LOADING}

The semi-cylindrical composite was bonded to the bone sample using a cyanoacrylate (superglue) adhesive and placed within the drive coil (Figure 6.1). Strain measurements were made using strain gages placed on the Terfenol-D composite surface, the bone surface adjacent to the composite, and the bone surface opposite the composite (Figure 6.2).

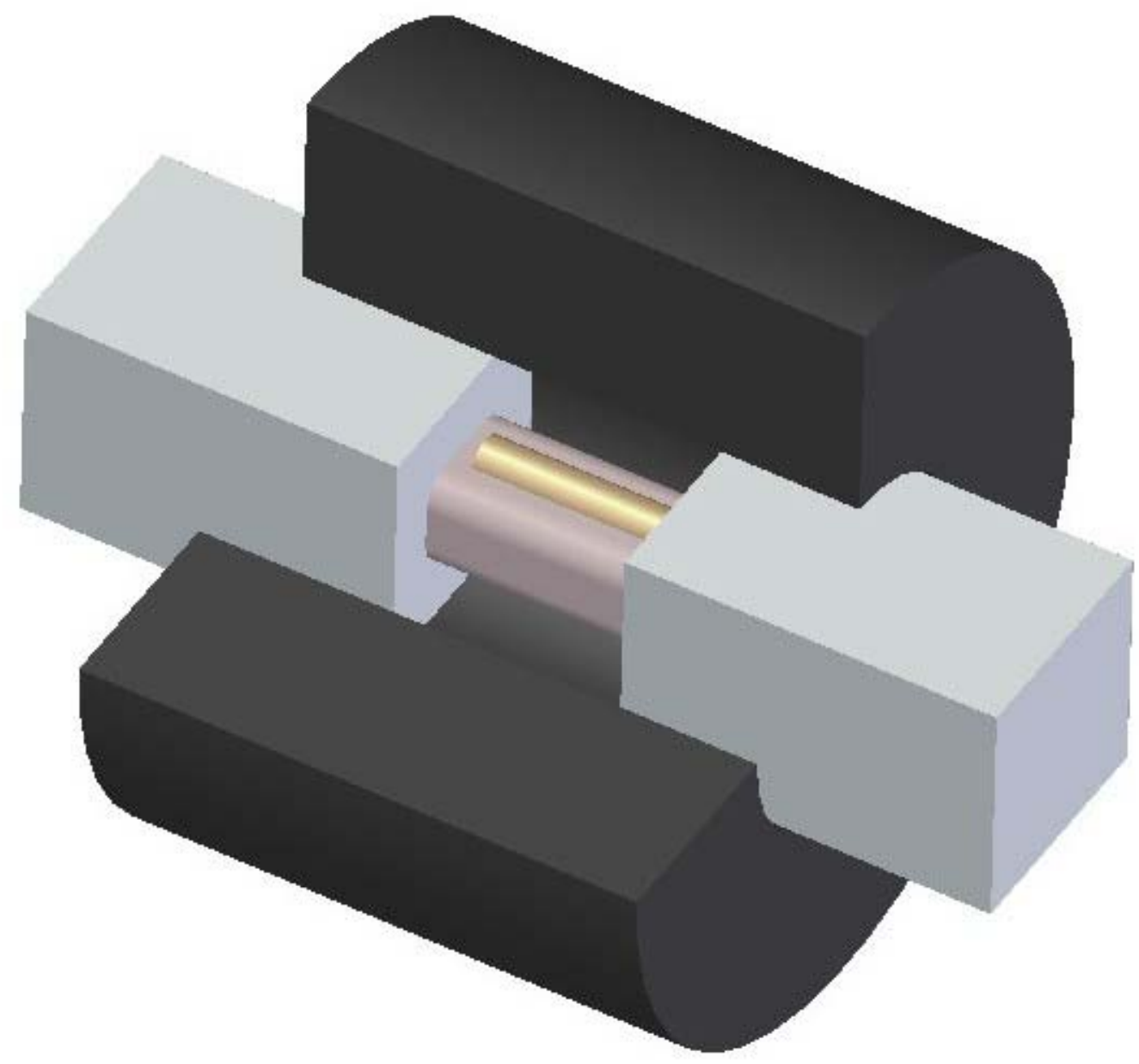

Figure 6.1: Experimental setup showing a cutaway of the drive coil (black), the Terfenol-D composite (gold), the bone sample (dark grey), and the aluminum mounting blocks (light grey) 


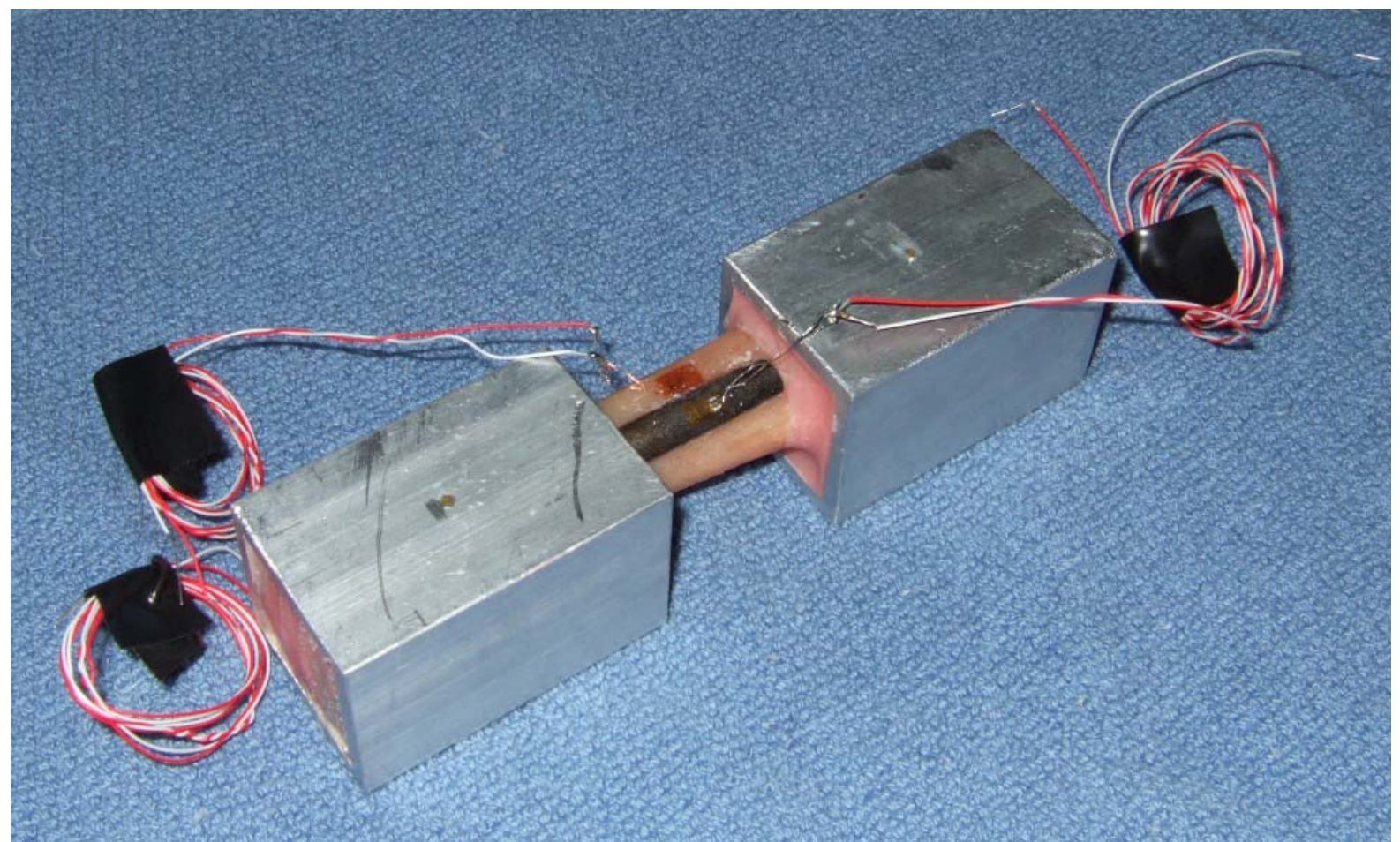

Figure 6.2: Tibia/Terfenol-D composite system showing strain gage measurements on the Terfenol-D composite surface and bone surface adjacent to the composite

Prior to bonding, initial dynamic tests of the Terfenol-D composite were conducted at fields ranging from 110 to $170 \mathrm{kA} / \mathrm{m}$ at a frequency of $30 \mathrm{~Hz}$. The results of these tests are shown in Figure 6.3. As the field intensity is increased, the strain produced in the Terfenol-D composite also increases up to a maximum of $2300 \mu \varepsilon$ peak to peak. The increasing trend in magnitude indicates that the saturation strain or maximum strain possible was not achieved. Increasing the field beyond $170 \mathrm{kA} / \mathrm{m}$ would produce increased strain magnitude up to the saturation strain of the material. Increased field beyond $170 \mathrm{kA} / \mathrm{m}$ was not possible at $30 \mathrm{~Hz}$ due to excessive heating of the drive coil. 


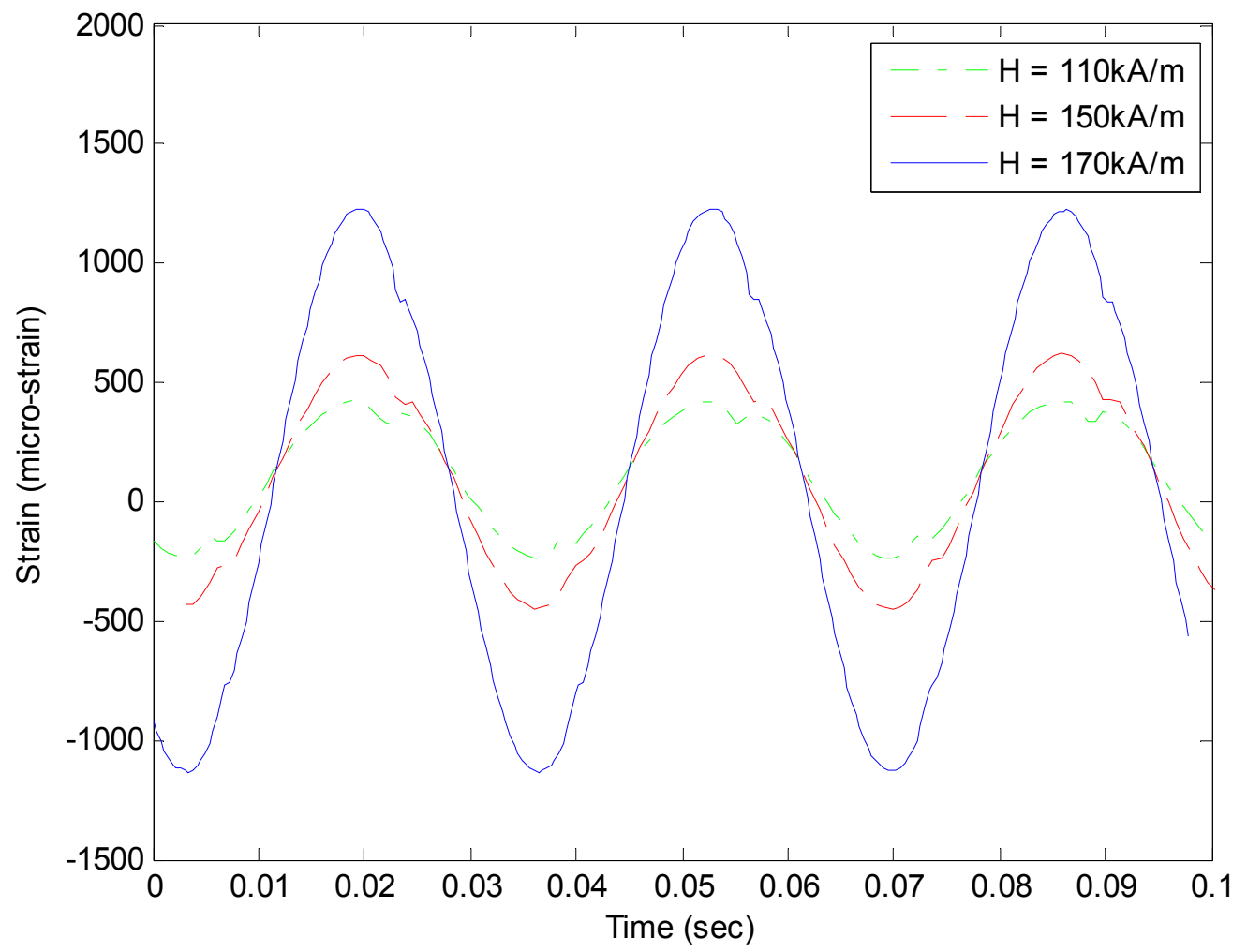

Figure 6.3: Unconstrained dynamic tests at $\mathrm{f}=30 \mathrm{~Hz}$ with varying field intensity

Once the unconstrained response of the composite was known, it was bonded to the bone for further testing. The composite was then driven at $\mathrm{f}=30 \mathrm{~Hz}$ at the highest field possible, $\mathrm{H}=170 \mathrm{kA} / \mathrm{m}$. The response of the composite under loaded (bonded) and free (unbonded) conditions is shown in Figure 6.4. 


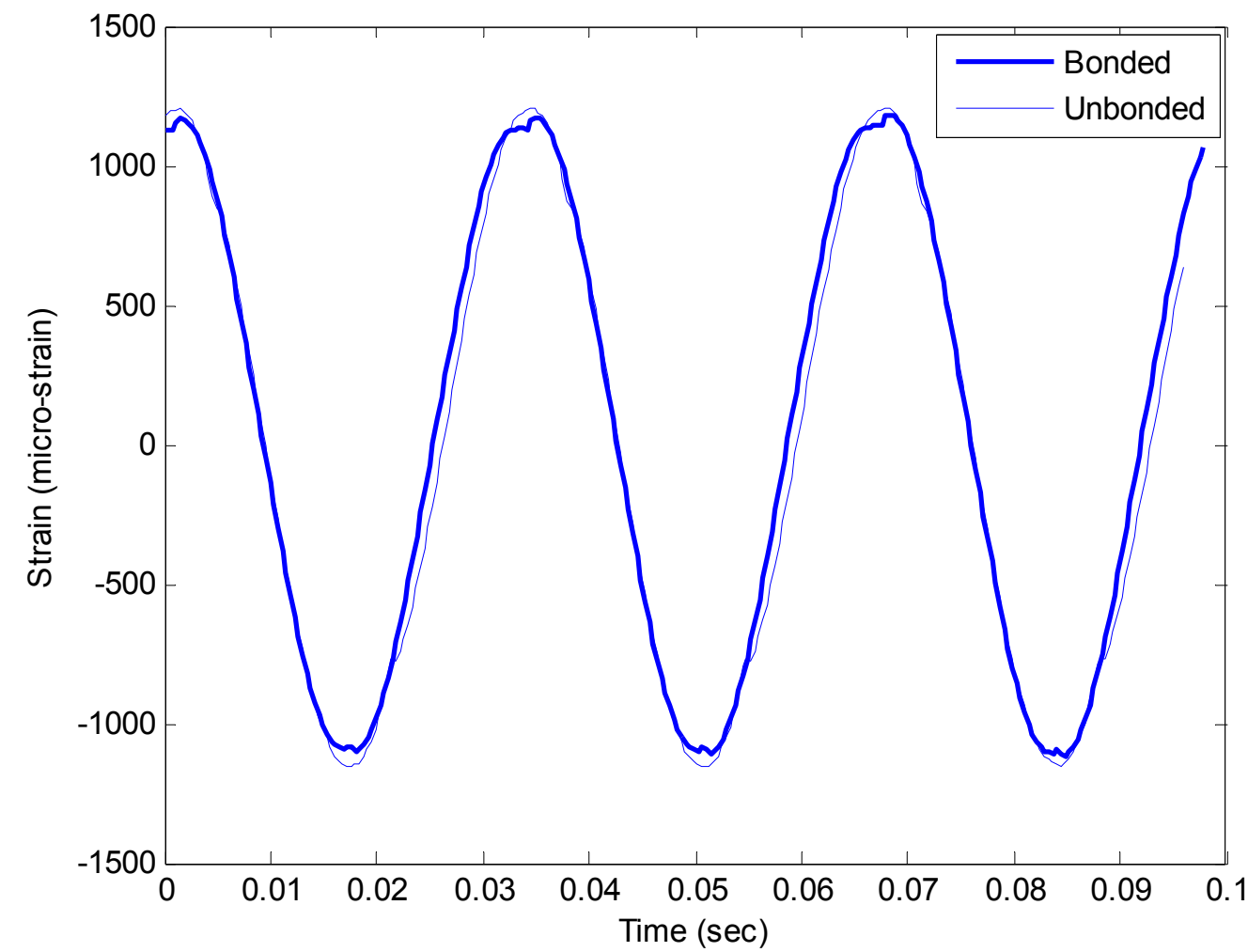

Figure 6.4: Strain magnitude for bonded and unbonded Terfenol-D composite at $H=170 \mathrm{kA} / \mathrm{m}$ and $\mathbf{f}=\mathbf{3 0 H z}$

Additional strain measurements were taken (Figure 6.5) on the composite/bone system on the bone surface adjacent to and opposite from the side bonded to the composite. The strain measurement on the surface of the composite is shown as the top graph in the figure, followed by the strain measurement on the bone surface adjacent to the composite, and finally the strain measurement on the bone surface opposite to the composite. The strain magnitude achieved by the Terfenol-D composite was greater than $2000 \mu \varepsilon$, however the bone surface was only subjected to $1000 \mu \varepsilon$. This loss in strain transmission was due to the viscoelastic properties of the adhesive used to bond the composite to the bone surface.

Despite the losses across the adhesive layer, the strain level achieved on the bone surface would be sufficient to promote the anabolic response of growth in accordance with previous research (Section 1.2.2). It is also noted that the strain level on both the top and bottom of the bone surface are approximately equal, meaning that the bone growth would 
theoretically occur at the same rate around the cross section of the bone with only one application point of the composite.
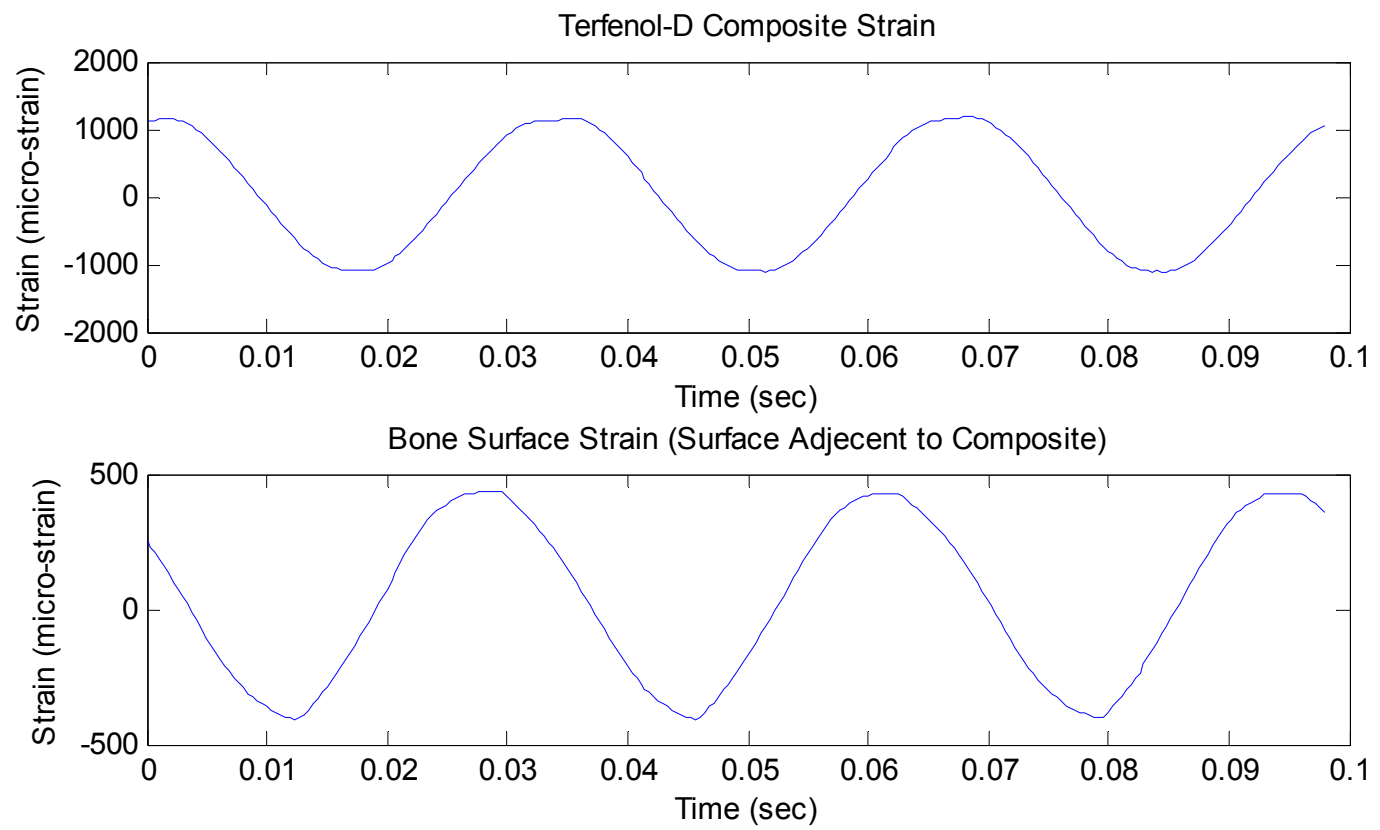

Bone Surface Strain (Surface Opposite to Composite)

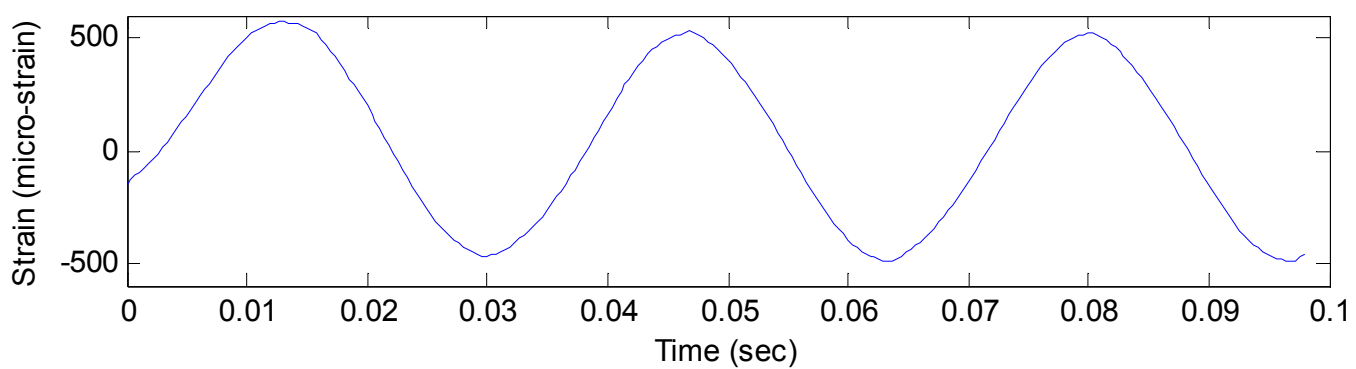

Figure 6.4: Dynamic bone test results showing Terfenol-D composite strain (top), bone surface strain adjacent to the composite (middle), and bone surface strain opposite to the composite (bottom).

As Figure 6.4 shows, the maximum pk-pk strain produced on the bone surface was approximately $1000 \mu \varepsilon$ at a frequency of $30 \mathrm{~Hz}$. This is sufficient strain magnitude and frequency to promote cortical bone growth in both rats and turkeys, and maintain cortical bone structure in humans.

\section{ONGOING INVESTIGATION}

Two questions remain to be answered. First, clarification of the pre-stress analysis is necessary and second, biocompatibility of the device needs to be resolved. To begin to answer these questions, a second Terfenol-D composite has been cast and will undergo a 
more accurate pre-stress testing using a force sensor with greater repeatability than the Flexiforce sensor.

Once a suitable biocompatible material is chosen to encapsulate the sample the next step is to perform in vivo testing on the tibia, ulnae, or other long bones of rats. Bone growth could be measured by administering intraperitoneal injections of calcein, which effectively label periosteal bone surfaces both before and after the loading regimen. Following tests, digital histomorphometry software could be utilized to determine the amount of bone growth achieved. 


\section{APPENDIX A}

\section{MIXING AND CASTING OF TERFENOL-D COMPOSITE}

\section{Materials:}

Derakane 411-C-50 Resin

Dow Chemical Co. of Channahon, IL

NOROX MEKP-925H

Norac, Inc. of Azusa, CA

Cobalt Napthenate, $6 \%$

The Shepherd Chemical Co. of Norwood, OH

Terfenol-D powder, 106-300 microns

ETREMA Products, Inc of Ames, Iowa

Silicon Rubber Sealant

DAP, Inc. of Baltimore, MD

Casting Mold

Permanent Magnet

Metal Mixing Container

Metal Table Spoon

Digital Scale

Guide Dowel

\section{List of Variables:}

$\begin{array}{ll}V_{M} & \text { Estimated total mixture volume }(\mathrm{mL}) \\ v f_{T} & \text { Desired volume fraction of Terfenol-D composite } \\ m_{T R} & \text { Mass of Terfenol-D powder required }(\mathrm{g}) \\ m_{E R} & \text { Mass of epoxy required }(\mathrm{g}) \\ m_{C} & \text { Mass of mixing container }(\mathrm{g}) \\ m_{\text {Resin }} & \text { Actual mass of resin }(\mathrm{g}) \\ m_{M E K P} & \text { Actual mass of MEKP }(\mathrm{g}) \\ m_{\text {CoNap }} & \text { Actual mass of CoNap }(g) \\ m_{E} & \text { Actual mass of epoxy }(g) \\ m_{T} & \text { Actual mass of Terfenol-D powder }(\mathrm{g}) \\ V F_{T} & \text { Actual volume fraction of Terfenol-D composite achieved }\end{array}$




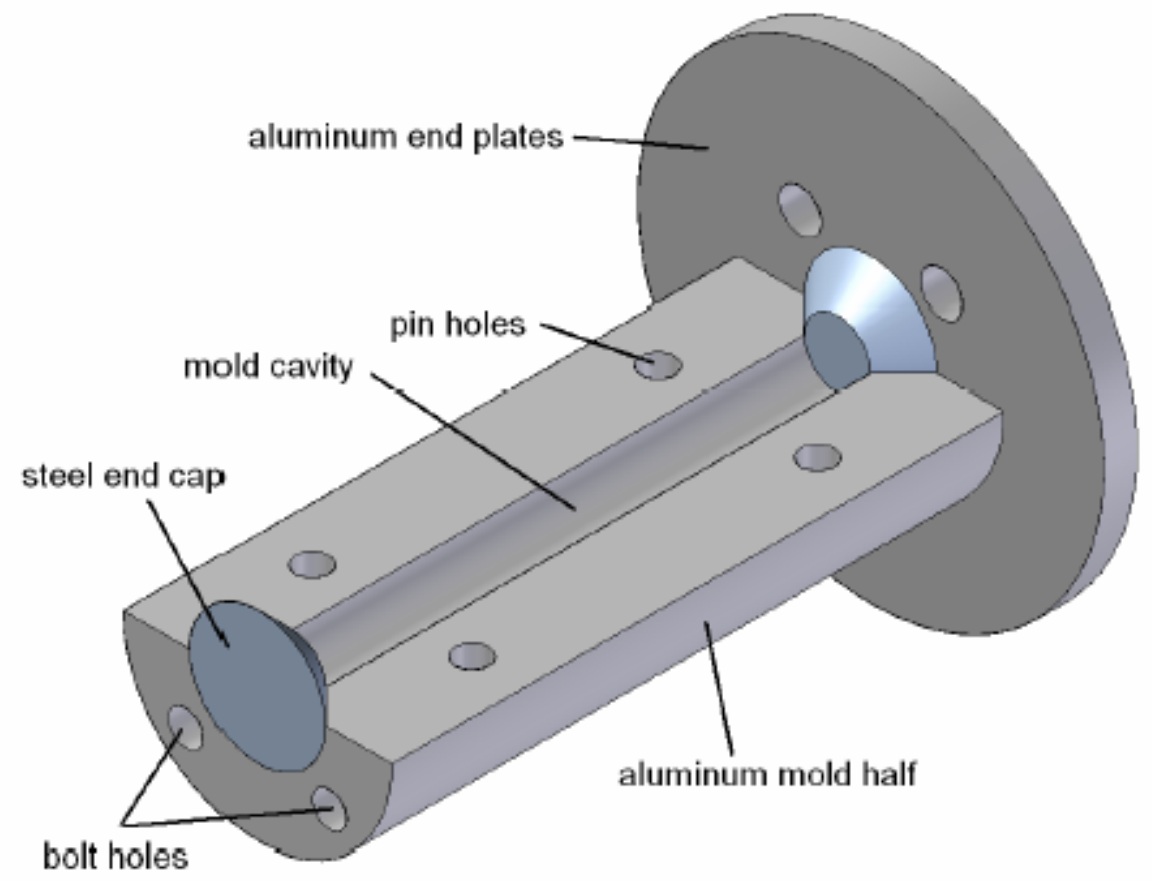

Figure A.1: Casting mold with top mold half and end plate removed [11]

\section{Procedure:}

1) Prepare the mold by sealing the mating surfaces of the mold halves and end caps with silicon rubber sealant.

2) Build the mold by mating the mold halves and placing one end cap in position. Secure this end cap by bolting one end plate in position and finger tighten the bolts.

3) Determine the amount of materials necessary to cast the composite. Measure the total mixture volume $\left(V_{M}\right)$ in cubic centimeters or milliliters by taking the mold volume and adding an additional factor to account for material left over in the mixing container.

(For a mold volume of $1.61 \mathrm{~mL}$, a total volume of $4 m L$ was typically mixed to achieve a $50 \%$ volume fraction of Terfenol-D.)

4) Choose a desired volume fraction of Terfenol-D $\left(v f_{T}\right)$. It is difficult to ensure proper wetting of Terfenol-D powder for volume fractions above $50 \%$.

5) From the mixture volume and volume fraction, estimate the mass of Terfenol-D powder required $\left(m_{T R}\right)$ and epoxy required $\left(m_{E R}\right)$ in grams by multiplying the volumes by the material densities. 


$$
\begin{aligned}
& m_{T R}=9.21 \frac{g}{m L}\left(v f_{T} \times V_{M}\right) m L \\
& m_{E R}=1.045 \frac{g}{m L}\left(\left(1-v f_{T}\right) \times V_{M}\right) m L
\end{aligned}
$$

6) Once adequate amounts of Terfenol-D and epoxy are acquired, measure and record the mass of the metal mixing container $\left(m_{C}\right)$ in grams.

7) Add $\left(m_{E R}\right)$ of Derakane resin to the mixing container. One tablespoon of resin is approximately $5 \mathrm{~g}$.

8) Calculate and record the actual mass of resin in the mixing container $\left(m_{\mathrm{Resin}}\right)$.

9) Calculate and record the mass of MEKP $\left(m_{M E K P}\right)$ in grams and CoNap $\left(m_{\text {CoNap }}\right)$ in grams required for the epoxy.

$$
\begin{aligned}
& m_{M E K P}=m_{\operatorname{Resin}} \frac{0.02}{0.978} \\
& m_{\text {CoNap }}=m_{\operatorname{Resin}} \frac{0.002}{0.978}
\end{aligned}
$$

10) Add the calculated amounts of MEKP and CoNap to the mixing container and mix thoroughly. About 30 minutes of working time is available for steps 11 through 19 before the epoxy begins to gel.

11) Calculate and record the actual amount of epoxy used $\left(m_{E}\right)$ by reading the total mass of the epoxy and mixture container and subtracting the mass of the mixing container measured in step 4.

12) Recalculate the mass of Terfenol-D powder in grams required to achieve the desired volume fraction based on the actual amount of epoxy mixed.

$$
m_{T R}=m_{E}\left(\frac{v f_{T}}{1-v f_{T}}\right)\left(\frac{9.21}{1.045}\right)
$$

13) Slowly add the calculated amount of Terfenol-D required mixing throughout the addition.

14) Calculated and record the actual amount of Terfenol-D added $\left(m_{T}\right)$ to the mixing container.

15) Once the mixture is homogenous, pack the mixture into the mold.

16) Place the remaining end cap in place, and slide the magnet guide dowel into position. 
17) Drop the permanent magnet down the guide dowel in a single motion.

18) Remove the guide dowel and finger tighten the remaining end plate into position.

19) Bake the mold and composite in a convection oven at $70^{\circ} \mathrm{C}$ for 6 hours to ensure a complete cure.

20) After the mold has cooled, remove one end plate and the permanent magnet. Tighten the bolts on the other end plate to "crack" the mold.

21) Remove the remaining bolts and dismantle the mold.

22) Remove all silicon from the mold and coat the mold surface with a thin layer of wax to preserve the mold.

23) Calculate and record the final volume fraction of Terfenol-D achieved in the composite.

$$
V F_{T}=\frac{m_{T} / 9.21}{m_{T} / 9.21^{+} m_{E} / 1.045}
$$




\section{APPENDIX B}

\section{FLEXIFORCE CALIBRATION CURVE AND CIRCUIT DIAGRAM}

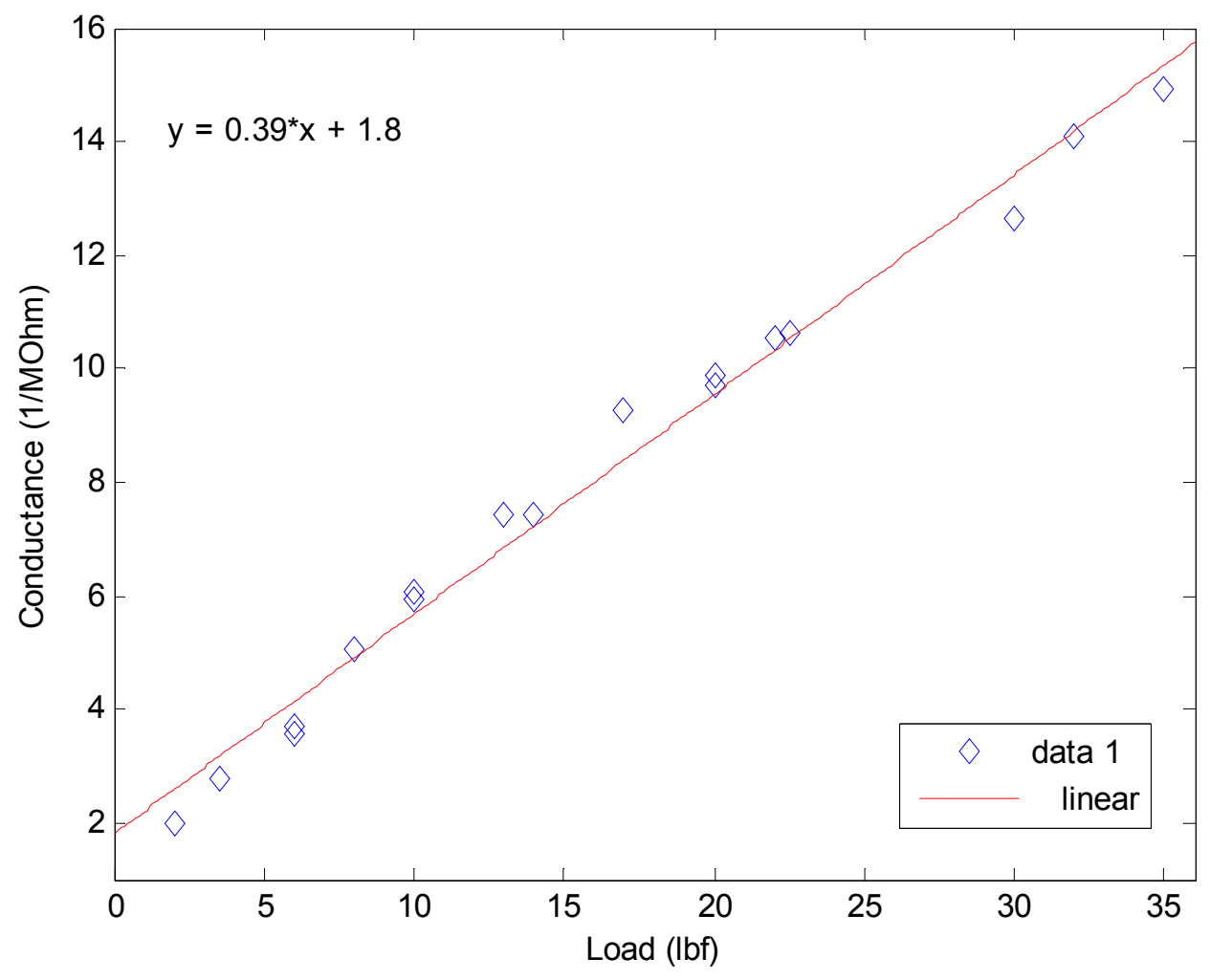

Figure B.1: FlexiForce calibration curve 


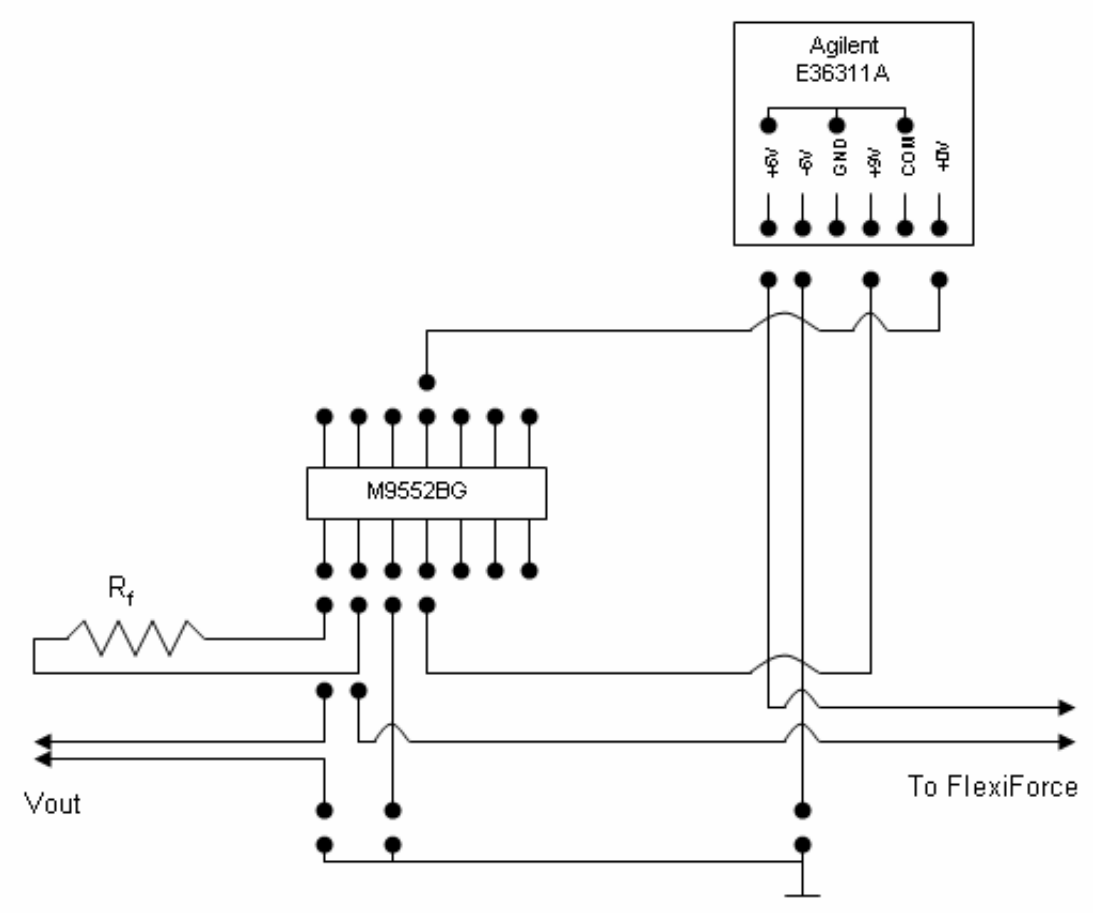

Figure B.2: Flexiforce breadboard schematic

The FlexiForce sensor changes resistance as a function of applied loads. In order to acquire this signal as a voltage, the sensor was placed in an inverting op-amp circuit shown in Figure B.2. The governing relation for the circuit is

$$
V_{\text {out }}=-\left(\frac{R_{f}}{R_{f f s}}\right) V_{e}
$$

where $V_{\text {out }}$ is the output voltage, $R_{f}$ is the feedback resistance, $R_{f f s}$ is the resistance of the FlexiForce sensor, and $V_{e}$ is the excitation voltage. If $R_{f}$ is set to the maximum $R_{f f s}$, then the output voltage will vary linearly between 0 and $V_{e}$ as $R_{f f s}$ changes. $\mathrm{R}_{\mathrm{f}}$ was set to $64.5 \mathrm{k} \Omega$ which corresponds to a load of $35.1 \mathrm{lbf}$ and $V_{e}$ was set to $+6 \mathrm{~V}$. 


\section{BIBLIOGRAPHY}

[1] Aarden, E.M., E.H. Burger, and P.J. Nijweide. "Function of osteocytes in bone." Journal of Cellular Biochemistry 55:287-299, 1994.

[2] Bilezikian, J.P., L.G. Raisz, and G.A. Rodan, Ed. "Principals of Bone Biology.” Academic Press, San Diego CA, 1996.

[3] Burger, E.H., and J. Klein-Nulend. "Mechanotransduction in bone - role of the lacuno-canalicular network." Federation of American Societies for Experimental Biology 13:S101-S112, 1999.

[4] Duenas, T.A. and G.P. Carmen. "Particle distribution study for low-volume fraction magnetostrictive composites." Journal of Applied Physics, 90:2433-2439, 2001.

[5] Duncan, R.L., and C.H. Turner. "Mechanotransduction and the functional response of bone to mechanical strain." Calcified Tissues International 21:211-214, 1995.

[6] Frost, H.M. "Dynamics of bone remodeling." Bone Biodynamics. Boston, Little, Brown 1964: 286292.

[7] Frost, H.M. "The mechanostat: A proposed pathogenically adaptive bone remodeling." Journal of Biomechanics 2:73-85, 1987.

[8] Liskova, M. and J. Hert. "Reaction of bone to mechanical stimuli. Part 2. Periosteal and endosteal reaction to tibial diaphysis in rabbit to intermittent loading." Folia Morphoogical 19:301-317, 1971.

[9] Martin, R.B. and D.B. Burr. "The Structure, Function and Adaptation of Compact Bone." Raven, New York, 1989.

[10] McKnight, G.P. [112] Oriented Terfenol-D composites. PhD thesis, University of California Los Angeles, CA, 2003.

[11] Mortensen, A.P. Characterization, modeling, and dynamic implementation of Terfenol-D particulate composites.MS thesis, The Ohio State University, OH, 2005.

[12] Khan, K., H. McKay, P. Kannus, D. Bailey, J. Wark, and K. Bennell. "Physical Activity and Bone Health.” Human Kinetics, Champaign IL, 2001.

[13] Kvarnsho, L. "On characterization, modeling, and application of highly magnetostrictive materials." Royal Institute of Technology, Stockhom, Sweeden, 1993.

[14] O’Connor, J.A., L.E. Lanyon, and J. MacFie. "The influence of strain rate on adaptive bone remodeling." Journal of Biomechanics 15:767-781, 1982.

[15] Owan, I., D.B. Burr, C.H. Turner, J. Qiu, Y. Tu, J.E. Onyia, and R.L. Duncan. "Mechanotransduction in bone: Osteoblasts are more responsive to fluid forces than mechanical strain." American Journal of Physiology 273:C810-C815, 1997.

[16] Robling, A.G., D.B. Burr, and C.H. Turner. "Partitioning a daily mechanical stimulus into discrete loading bouts improves the osteogenic response to loading." Journal of Bone and Mineral Research 15:1595-1602, 2000. 
[17] Robling, A.G., D.B. Burr, and C.H. Turner. "Recovery periods restore mechanosensitivity to dynamically loaded bone.” Journal of Experimental Biology 204:3389-3399, 2001.

[18] Rubin, C.T., A.S. Turner, C. Millinckrodt, C. Jerome, K. Mcleod, and S. Bain. "Mechanical strain, induced noninvasively in the high-frequency domain, is anabolic to cancellous bone, but not cortical bone." Bone 30:445-452, 2002.

[19] Rubin, C.T. and L.E. Lanyon. "Regulation of bone formation by applied dynamic loads." Journal of Bone and Joint Surgery 66-A:397-402, 1984.

[20] Rubin, C.T. and L.E. Lanyon. "Regulation of bone mass by mechanical strain magnitude." Calcified Tissues International 37:411-417, 1985.

[21] Rubin, C.T., R. Recker, D. Cullen, J Ryaby, J. McCabe, and K. McLeod. "Prevention of postmenopausal bone load by a low-magnitude, high frequency mechanical stimuli: A clinical trial assessing compliance, efficacy, and safety." Journal of Bone and Mineral Research 19:343-351, 2004.

[22] Sanders, J.O., A.E. Sanders, R. More, and R.B. Ashman. "A Preliminary investigation of shape memory alloys in the surgical correction of scoliosis." Spine 18:1640-1646, 1993.

[23] Saxon, L.K., A.G. Robling, L. Alam, and C.H. Turner. "Machanosensitivity of the rat skeleton decreases after a long period of loading, but is improved with time off." Bone 36:454-464, 2005.

[24] Schmerling, M.A., M. A. Wilkov, A.E. Sanders, and J.E. Woosley. "Using the Shape Recovery of Nitinol in the Harrignton Rod Treatment of Scoliosis." Journal of Biomedical Materials Research 10:876-892, 1976.

[25] Srinivasan, S. and T.A. Gross. "Intermittent rest enhances osteoblastic activation induced by mechanical loading." Transactions of the Orthopedic Research Society 25:628, 2000.

[26] Srinivasan, S., D.A. Weimer, S.C. Agans, S.D. Bain, and T.S. Gross. "Low-magnitude loading becomes osteogenic when rest is inserted between each load cycle." Journal of Bone and Mineral Research 17:1613-1620, 2002.

[27] Thompson, D.W. and J.T. Bonner, Ed. "On Growth and Form." Cambridge: Cambridge University, 1961.

[28] Turner, C.H. “Three Rules for Bone Adaptation to Mechanical Stimuli." Bone 23: 399-407, 1998.

[29] Turner, C.H., M.R. Forwood, and M.W. Otter. "Mechanotransduction in bone: Do bone cells act as sensors of fluid flow?" The Federation of American Societies for Experimental Biology Journal 8:875-878, 1994.

[30] Turner, C.H., Forwood, M.R., Rho, J.Y., and Yoshikawa, T. "Mechanical loading thresholds for lamellar and woven bone formation." Journal of Bone and Mineral Research 9:87-97, 1994.

[31] Turner, C.H., I. Owan, and Y. Takano. "Mechanotransduction in bone: Role of strain rate." American Journal of Physiology 269:E438-E442, 1995.

[32] Umemura, Y., T. Ishiko, T. Yamauchi, M. Kurono, and S. Mashiko. "Five jumps per day increases bone mass and breaking force in rats." Journal of Bone and Mineral Research 12:1480-1485, 1997.

[33] Wolff, J. “Das Gesetz der Transformation der Knochen.” Hirschwald, Berlin, 1892. 